\title{
Indoor Localization and Radio Map Estimation using Unsupervised Manifold Alignment with Geometry Perturbation
}

\author{
Khaqan Majeed, Sameh Sorour, Senior Member, IEEE, Tareq Y. Al-Naffouri, Member, IEEE, \\ and Shahrokh Valaee, Senior Member, IEEE
}

\begin{abstract}
The Received Signal Strength (RSS) based fingerprinting approaches for indoor localization pose a need for updating the fingerprint databases due to dynamic nature of the indoor environment. This process is hectic and time-consuming when the size of the indoor area is large. The semi-supervised approaches reduce this workload and achieve good accuracy around $15 \%$ of the fingerprinting load but the performance is severely degraded if it is reduced below this level. We propose an indoor localization framework that uses unsupervised manifold alignment. It requires only $1 \%$ of the fingerprinting load, some crowd sourced readings and plan coordinates of the indoor area. The $1 \%$ fingerprinting load is used only in perturbing the local geometries of the plan coordinates. The proposed framework achieves less than $5 \mathrm{~m}$ mean localization error, which is considerably better than semi-supervised approaches at very small amount of fingerprinting load. In addition, the few location estimations together with few fingerprints help to estimate the complete radio map of the indoor environment. The estimation of radio map does not demand extra workload rather it employs the already available information from the proposed indoor localization framework. The testing results for radio map estimation show almost $50 \%$ performance improvement by using this information as compared to using only fingerprints.
\end{abstract}

Index Terms_-Indoor Localization, Radio Map Estimation, Manifold Alignment.

\section{INTRODUCTION}

The advent of mobile devices (e.g. laptops, personal digital assistants (PDAs), smart phones and tablets) has resulted in the proliferation of location-based services (LBS). The Global Positioning System (GPS) offers suitable accuracy in outdoor location estimation applications but fails to provide satisfactory performance in indoor environments due to the unpredictability of radio propagation. This is because the GPS signals sent from satellites to devices become very weak while penetrating through buildings. On the other hand, wireless Local Area Networks (WLANs) infrastructure using RSS has gained much importance recently over Angle-of-Arrival (AoA) and Time-of-Arrival (ToA)/Time-Difference-of-Arrival (TDoA) techniques [2][4]. The superiority of RSS based WLAN positioning has been stressed in [13], [14]. Some of the other techniques make use of sensory measurements [5]-[8] in users' mobile phones. The schemes employing Wi-Fi fingerprinting [9]-

- K. Majeed and S. Sorour are with the Department of Electrical Engineering, King Fahd University of Petroleum and Minerals (KFUPM), Dhahran, Saudi Arabia (e-mail: \{g201105490, samehsorour\}@kfupm.edu.sa)

- T. Y. Al-Naffouri is with the Department of Electrical Engineering, King Fahd University of Petroleum and Minerals (KFUPM), Dhahran, Saudi Arabia and also with the Department of Electrical Engineering, King Abdullah University of Science and Technology (KAUST), Thuwal 239556900, Saudi Arabia (e-mail: tareq.alnaffouri@kaust.edu.sa)

- S. Valaee is with Edward S. Rogers Sr. Department of Electrical and Computer Engineering, University of Toronto, 10 King's College Road, Toronto, ON, M5S 3G4, Canada (e-mail: valaee@comm.utoronto.ca)

- The authors would like to acknowledge the support provided by the Deanship of Scientific Research (DSR) at King Fahd University of Petroleum and Minerals (KFUPM) for funding this work through project No. FT131010.

- This work is an extension to our paper [1] in WCNC 2014.
[12], [30] do not require any investment cost in deploying Access Points (APs), i.e. existing installed APs can be used for location estimation. Moreover, the mobile devices are widely available nowadays and are equipped with Wireless Network Interface Controller (WNIC) so there is no need for additional hardware changes.

Indoor positioning using WLAN RSS is broadly divided into two categories. One category makes use of radio propagation models and statistical modeling as in [15]. The other category deals with location fingerprinting [9]-[12], [16], [30]. Location fingerprinting involves two phases, an offline phase and online one. The radio or fingerprinting map is obtained in the offline phase where RSS readings from concerned APs are collected at each reference or grid point in the indoor area. The online phase involves the collection of localization request(s) (RSS readings measured by user(s) to obtain their location(s)). Based on the amount of fingerprinting load, we can further divide the indoor localization schemes into two classes. One employing full fingerprinting map and the other using partial fingerprinting map to estimate the positions in an indoor area.

An example of full fingerprinting map based technique is [18], where the estimated position is obtained by taking centroid of the K nearest neighbors to the RSS reading of the localization request. The technique proposed in [10][12] employs compressive sensing [19]-[21] to localize user, where the user is considered as sparse in the spatial domain. The cross correlation information of the signals at different APs is taken into account in [16] for localization. The location estimation in [17] is done using TDoA and compressed sensing.

Practical implementation of fingerprinting based indoor 
localization systems requires greater workload in huge indoor areas since the RSS readings need to be collected at all specified grid points. Moreover, the indoor areas are also dynamic (for instance, moving carts, people, doors, elevators, and escalators). This acts as the main cause of RSS variations when measured at the same grid point. To reduce the need, cost and effort in constructing and updating full radio maps, which is a must for all the solutions described above, another set of solutions is employing learning methods to either estimate the radio map or localize using a limited number of fingerprints. One solution with reduced fingerprinting load is proposed in [30], which employs interpolation method for localization. Some solutions [5][8] estimate location by collecting readings from inertial sensors (gyroscope, compass, and accelerometer) present in the mobile devices. Semi-supervised manifold alignment (SMA) [23] is used in [28], [29] to localize users.

In this work, we aim to further reduce the fingerprinting load (1-5\%) while still maintaining low degradation in performance. In this approach, the server collects a considerable number of crowd sourced RSS readings from the casual visitors of the environment. Nonetheless, we still require some very limited number of labeled positions (fingerprints) to perturb the geometry of coordinates since the plan coordinates have symmetrical structure. Indeed, it becomes difficult to differentiate between the small chunk of points from one place to another in an indoor area. These few fingerprints are termed as calibration data/calibration readings.

Localization requests are obtained in the online phase and fall under the category of unlabeled data. These are the readings obtained from users who are interested in the estimation of their locations. Unsupervised Manifold Alignment (UMA) [25] is used to tackle the problem of feature matching between the collected readings and the available coordinate plan of the indoor environment. The non-bijective mapping functions transfer the information from the readings data set to the coordinates data set. The collection of RSS readings in the online phase takes care of temporal variations in them to a great extent.

Another task in indoor localization problem involves the estimation of radio coverage of Wi-Fi APs in an indoor area. The APs available in the indoor area are not accessible at all the locations. For example, for a large indoor area such as a university campus or an airport, the AP accessible at north side of the area may not be accessible at the south side. The estimation of signal strength from an AP in an indoor environment gives an idea of possible coverage throughout the region from that AP. The estimated signal strength values help predict performance of different services. For instance, VoIP services, video calling, and video streaming require high data rates, which in turn require good signal strength. For low data rate services like online browsing, and text chatting, low signal strength values are also acceptable. Moreover, it also helps to find replacement locations for APs in an indoor environment for good radio coverage. The placement of a new AP can be judged based on the already available radio map. The new placement of APs can also help to reduce the spillage of the signals outside the indoor premises. These useful applications pose a need to estimate radio coverage in an indoor environment. The estimated radio map can also be used to estimate new localization requests directly from the map by means of much simpler algorithms like nearest neighbor (NN) and k-nearest neighbors approaches. We propose a simple solution for estimating the complete radio map of the indoor environment which requires only few fingerprints, few localization requests and plan coordinates of the indoor area. The estimation of radio map from this information can be regarded as the by-product of the indoor localization system since it does not need any extra information.

The contributions of the proposed work can be summarized as following. The hectic phase of building radio map is eliminated because the fingerprinting load used for localization is almost negligible (approx. 1\% of the whole radio map). This helps in easy deployment of the indoor localization system in new indoor environments. The collection of crowd sourced readings at the time of localization or a short time before localization helps to combat temporal variations in RSS readings. Secondly, the fingerprints (approx. 1\%) together with few location estimations help to estimate the complete radio map of the whole indoor environment without any need of extra information.

The organization of the remaining portion of the paper is as follows: The description of the aforementioned prior works and comparison with our technique is presented in Section 2. Section 3 describes the problem setup for the indoor localization framework. This is followed by the indoor localization framework proposed in Section 4 . The testing results for the indoor localization framework are described in Section 5. The proposed methodology for radio map estimation is described in Section 6 and its testing results in Section 7. Finally the paper is concluded in Section 8.

\section{Related Work}

Several full fingerprinting map based techniques have been proposed. The simplest one gives the estimated position as the centroid of the K nearest neighbors to the RSS reading of the localization request [18]. Another solution proposed in [10], [12] makes use of compressive sensing [19]-[21]. These works treat the problem as sparse i.e. users are sparse in the spatial domain and compressive sensing is employed for location estimation. The effect of device orientation is also taken into account in [11], [12]. The problem is also treated as sparse in [16], in which the fingerprinting map is constructed by taking into account the cross-correlation information of the signals at different APs. The locations of the users are then reconstructed by solving an $l_{1}$-norm minimization problem. A similar solution methodology is proposed in [17] using TDoA and compressed sensing. Cooperation among APs is taken into account there to exploit cross-correlation of the signals at different APs. The configuration of the APs is known a priori along with the grid structure for localization. Moreover, this TDoA solution is a passive localization technique, in which the location of the mobile user is estimated without mobile phone's active involvement. However, the proposed solution in this work neither requires any information about the APs nor any communication between them. 
Among partial fingerprinting map based techniques, one solution proposed in [30] makes use of a limited number of fingerprints to estimate the positions. An interpolation function is used to give the $(x, y)$ coordinates as a function of RSS values. Another recent example employs semisupervised manifold alignment (SMA) [23] to solve the localization problem in the presence of a limited number of fingerprints [28], [29]. In semi-supervised localization approaches, a small percentage of the RSS fingerprints is obtained throughout the indoor area and are termed as labeled data (calibration information/data/readings). The RSS readings from users are obtained in the online stage for location estimation and are called unlabeled data (or localization requests). SMA then aligns the labeled and unlabeled data in a common lower dimensional space. The closest matches then provide the estimated positions. Good performance is achieved at a low fraction (15-30\%) of fingerprints and thus more studies are required to further reduce these percentages while achieving similar performances.

The only similarity between the proposed work and [29] is that they both try to reduce calibration (and even this to different levels) using a broad family of machine learning techniques called manifold alignment (and even in this family, we use two totally different sub-categories one semi-supervised and the other unsupervised). However, the assumptions, inputs to the problems and most importantly the employed techniques (with details) are totally different. The localization technique proposed in [29] makes use of semi-supervised manifold alignment whereas in the proposed work unsupervised manifold alignment is used. In [29] considerable number of fingerprints are collected in the offline phase for good performance. The collection and/or updating of fingerprints is a very hectic and time consuming process especially if the indoor area is huge. On the contrary, if the localization is performed by reducing the number of fingerprints, the performance is severely affected as mentioned in Section 5. This is because the semi-supervised manifold alignment (in [29]) requires considerable number of labeling positions to estimate the unlabeled positions with good accuracy. The unsupervised manifold alignment used in the proposed work requires no labeling information as opposed to the semi-supervised technique in [29]. The $1 \%$ labeling information (fingerprints) collected in our case are enough for perturbing the geometry of the plan coordinates since high symmetry exists in the plan coordinates. This improves the localization accuracy considerably as compared to the semi-supervised approach in [29] at the same level of fingerprinting load (calibration readings). The big difference in the inputs of the problem result in big difference in the employed algorithms in both cases. The readings data set in proposed work consists of fingerprints, crowd sourced readings and localization requests whereas in [29], it consists of fingerprints and localization requests. The number of fingerprints (calibration readings) used in [29] are very large as compared to the proposed work. The use of real-time (or quasi-real-time) crowd sourced information in the proposed work provides inherent updating of RSSs at time of localization. Whereas in [29] the large number of fingerprints need to be collected again for updating.

Another set of solutions [5]-[8] eliminate the workload by making use of inertial sensors (gyroscope, compass, and accelerometer) present in the mobile devices. Due to the internal structure of indoor area (people taking escalators, elevators, stairs or walking on the floor), the readings obtained from these sensors have a particular trend. These readings actually give a coarse localization of the user. The RSS readings along with the data gathered from inertial sensors then help to fine tune the estimation. The analysis of these techniques shows that still some time and resources are required for the practical deployment of such systems to obtain an acceptable level of localization accuracy. In addition, these techniques need to collect information from the inertial sensors present in smart phones, which, in fact, adds to the additional hardware requirements. For each new indoor area, some sort of training procedure is required in initial running of the algorithm, which is a bottleneck in a practical implementation of such systems.

The partial fingerprinting load along with user traces (crowd sourced information) is also considered in [31], [32] for location estimation. The percentage of fingerprints considered in [31], [32] is still high than the method we proposed. The user traces considered for performance improvement are obtained by collecting RSS readings from moving users in the indoor environment. Initial setup time and resources are still required for practical implementation of these systems in new indoor environments. However, in our proposed method, the $1 \%$ fingerprinting load is used only for perturbing the local geometries of the plan coordinates. And the crowd sourced information considered is also a small fraction of the total grid points, which can be obtained at the time of localization or a short time before localization from stationary users in the indoor environment.

\section{Problem formulation}

To set up the problem, some RSS readings are collected. These readings include few calibration readings (another name for fingerprints), some crowd sourced readings (RSS readings gathered from casual users walking in the indoor area), localization request(s) and indoor plan coordinates. The main point to note here is that a very small number of fingerprints (approximately $1 \%$ of the whole radio map) are used in this work. The collected RSS readings (calibration readings, crowd sourced readings and localization requests) and plan coordinates of the indoor area are arranged in the form of source and destination data sets. The unsupervised manifold alignment with geometry perturbation then aligns the data sets in a common lower dimensional (hyper)plane. Following notations are used to address different variables throughout the paper. Scalar by $m$, constant by $N$, matrix by $\mathbf{V}$, vector by $\mathbf{v}$, set by $\mathcal{X}, i^{t h}$ member of a set $\mathcal{X}$ (vector case) by $\mathbf{x}_{i}, i^{\text {th }}$ member of a set $\mathcal{X}$ (scalar case) by $x_{i}$, and $k^{\text {th }}$ dimension of $i^{\text {th }}$ member of a set $\mathcal{X}$ by $x_{k}{ }^{(i)}$.

The indoor area is discretized into $N$ grid points or positions with some fixed distance in-between the points. The fingerprints are obtained at these points (in our case as low as $1 \%$ of the total points). The calibration readings or fingerprints are obtained in the offline phase and crowd sourced readings and localization requests in the online phase. Since a very small number of fingerprints is used for location estimations in our case so these fingerprints can 
also be collected in the online phase by the use of sniffers ${ }^{1}$. Another advantage is that online fingerprinting would be more accurate because it is not outdated. This eliminates the workload completely to such an extent that the database of these very few fingerprints can be updated continuously without the aid of manpower. The following subsections describe the construction of source and destination data sets.

\subsection{Source Data Set}

The source data set $\mathcal{X}$ contains $R$-dimensional vectors representing the RSS readings measured from $R$ APs. The set is constructed by concatenating the calibration or fingerprinted readings set $\mathcal{C}$, the crowd sourced readings set $\mathcal{S}$ and the localization requests set $\mathcal{L}$. Note that the calibration readings are readings obtained at known locations whereas crowd sourced readings are received from unknown locations. Consider the calibration readings set $\mathcal{C}$. Each element $\mathbf{c}_{i}$ of $\mathcal{C}$ is a vector of RSS measurements obtained at a known position $i$ in the spatial domain from $R$ APs. In other words $\mathbf{c}_{i}=\left[\begin{array}{llll}c_{1}^{(i)} & c_{2}^{(i)} & \cdots & c_{R}^{(i)}\end{array}\right]^{T}$. We can thus describe $\mathcal{C}$ as

$$
\begin{aligned}
\mathcal{C} & =\left\{\mathbf{c}_{1}, \mathbf{c}_{2}, \cdots, \mathbf{c}_{f}\right\} \\
& =\left\{\left[\begin{array}{c}
c_{1}^{(1)} \\
c_{2}^{(1)} \\
\vdots \\
c_{R}^{(1)}
\end{array}\right],\left[\begin{array}{c}
c_{1}^{(2)} \\
c_{2}^{(2)} \\
\vdots \\
c_{R}^{(2)}
\end{array}\right], \cdots,\left[\begin{array}{c}
c_{1}^{(f)} \\
c_{2}^{(f)} \\
\vdots \\
c_{R}^{(f)}
\end{array}\right]\right\}
\end{aligned}
$$

These fingerprints are obtained at $f$ grid points ${ }^{2}$, which, as indicated earlier, are very small in number as compared to the total grid points of the indoor area under consideration (that is, $f \ll N$ ). Similarly, the elements presented in crowd sourced readings set $\mathcal{S}$ and the localization requests set $\mathcal{L}$ also contain RSS readings, in vector form, from $R$ APs. The $i^{t h}$ element of these sets is given by, respectively, $\mathbf{s}_{i}=\left[\begin{array}{cccc}s_{1}^{(i)} & s_{2}^{(i)} & \cdots & s_{R}^{(i)}\end{array}\right]^{T}$ and $\mathbf{l}_{i}=\left[\begin{array}{llll}l_{1}^{(i)} & l_{2}^{(i)} & \ldots & l_{R}^{(i)}\end{array}\right]^{T}$. Thus, the sets $\mathcal{S}$ and $\mathcal{L}$ are described by

$$
\begin{aligned}
& \mathcal{S}=\left\{\left[\begin{array}{c}
s_{1}^{(1)} \\
s_{2}^{(1)} \\
\vdots \\
s_{R}^{(1)}
\end{array}\right],\left[\begin{array}{c}
s_{1}^{(2)} \\
s_{2}^{(2)} \\
\vdots \\
s_{R}^{(2)}
\end{array}\right], \cdots,\left[\begin{array}{c}
s_{1}^{(o)} \\
s_{2}^{(o)} \\
\vdots \\
s_{R}^{(o)}
\end{array}\right]\right\} \\
& \mathcal{L}=\left\{\left[\begin{array}{c}
l_{1}^{(1)} \\
l_{2}^{(1)} \\
\vdots \\
l_{R}^{(1)}
\end{array}\right],\left[\begin{array}{c}
l_{1}^{(2)} \\
l_{2}^{(2)} \\
\vdots \\
l_{R}^{(2)}
\end{array}\right], \cdots,\left[\begin{array}{c}
l_{1}^{(r)} \\
l_{2}^{(r)} \\
\vdots \\
l_{R}^{(r)}
\end{array}\right]\right\}
\end{aligned}
$$

1. Sniffers are hardware or computer programs that log or intercept traffic over wireless or Ethernet Local Area Network (LAN).

2. There is no strict criterion for the selection of fingerprints in an indoor area. The only requirement is that the chosen fingerprints are well apart and are not located in constricted portion of the area. Consider the formation of imaginary circles with the selected fingerprints as centers of the circles. Following conditions should be fulfilled for these circles. (1) The circles should have almost the same radii, (2) the adjacent circles should form a minimum overlapping set of coordinates with each other, and (3) the circles should cover the whole coordinate plan.
The structure of the source data set $\mathcal{X}$ is thus

$$
\mathcal{X}=\left\{\mathbf{c}_{1}, \cdots, \mathbf{c}_{f}, \mathbf{s}_{1}, \cdots, \mathbf{s}_{o}, \mathbf{l}_{1}, \cdots, \mathbf{l}_{r}\right\}
$$

The total number of elements in $\mathcal{X}$ is $M=f+o+r$. As noticed, the set $\mathcal{X}$ is comprised of three portions. The first portion of the set $\mathcal{X}$, which represents the calibration readings or fingerprints, is not used in unsupervised manifold alignment but rather in perturbing the local geometries of the indoor plan coordinates. This will become clear in Section 4.

\subsection{Destination Data Set}

The destination data set $\mathcal{Z}$ is formed using 2-dimensional coordinates corresponding to the physical locations in the indoor area. These elements are arranged in such a manner that the first $f$ coordinate pairs are in correspondence with the calibration readings and remaining portion of the set contains the remaining coordinates of the indoor area. Thus, we can write

$$
\mathcal{Z}=\left\{\mathbf{p}_{1}, \cdots, \mathbf{p}_{f}, \mathbf{p}_{f+1}, \cdots, \mathbf{p}_{N}\right\}
$$

where, the $i^{t h}$ element represents the $i^{\text {th }}$ coordinate pair and is given by $\mathbf{p}_{i}=\left[\begin{array}{l}x^{(i)} \\ y^{(i)}\end{array}\right]$.

The cardinality of the set $\mathcal{Z}$ is $N$. The main point to note here is that $M \neq N$, which necessarily implies that $(o+r) \neq(N-f)$. Since the RSS readings are taken at some coordinates so $M$ is usually much less than $N$. Figure 1 shows the structure of the source and destination data sets.

The output of the algorithm provides estimated positions of the last $r$ readings or localization requests in the set $\mathcal{X}$. That is,

$$
\hat{\mathcal{P}}_{\mathcal{L}}=\left\{\hat{\mathbf{p}}_{l_{1}}, \hat{\mathbf{p}}_{l_{2}}, \cdots, \hat{\mathbf{p}}_{l_{r}}\right\}
$$

These estimated positions with associated RSS readings help in estimating the radio map in the indoor area. This is described in Section 6.

\section{Unsupervised Manifold Alignment}

Unlike its semi-supervised counterpart [23], unsupervised manifold alignment [25] is a transfer learning technique

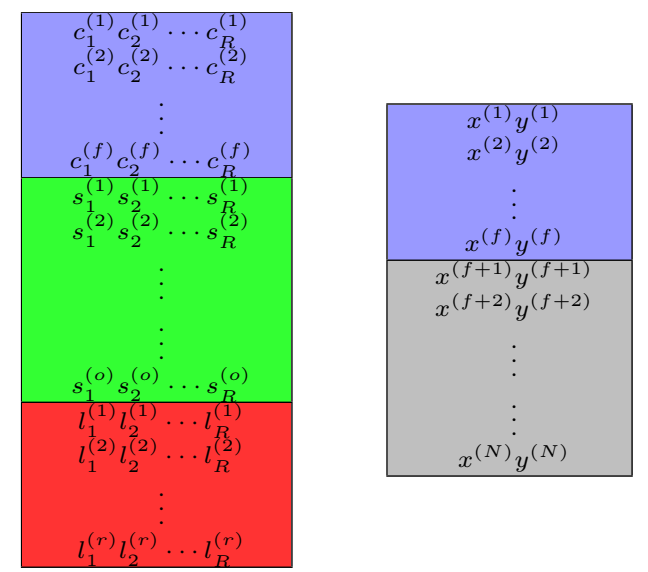

Fig. 1. Structure of Source (left) and Destination (right) data sets 
that does not need any correspondence information to align the data sets in a lower dimensional space. It replaces the considerable percentage of labeled data (in our case fingerprinted RSSs) required for the semi-supervised approach by unlabeled data (in our case crowd sourced RSSs). To perform manifold alignment in such an unsupervised environment, the similar correlation patterns of the source and destination data sets (i.e. the fact that neighboring points have stronger correlation as compared to distant points) is exploited. This assumption allows the matching of intrinsic structures between two data sets. Instead of performing a transformation of the data sets to a common lower dimensional space, the source data set is transformed by a non-bijective mapping function to the destination data set. The perfect matchings are then obtained by smallest distances in a mutual embedding space. In aligning the two data sets in a common lower dimensional space, it is important to maintain the following: Preservation of local geometries within each data set, and matching of local geometries between the two data sets.

Consider our two sets, the source set $\mathcal{X}$ with $M$ elements

$$
\mathcal{X}=\left\{\mathbf{x}_{1}, \mathbf{x}_{2}, \cdots, \mathbf{x}_{M}\right\}
$$

and the destination set $\mathcal{Z}$ with $N$ elements

$$
\mathcal{Z}=\left\{\mathbf{z}_{1}, \mathbf{z}_{2}, \cdots, \mathbf{z}_{N}\right\}
$$

The perfect geometry matchings are obtained between these data sets by simultaneously preserving the local geometries in each set. The following subsections describe the above mentioned points of aligning data for this specific application.

\subsection{Geometry Perturbation of Destination Data Set}

As stated earlier, the destination data set used in the manifold alignment problem is constructed using plan coordinates of the indoor area. The important point in manifold alignment is the matching of local geometries between the sets, so within a data set, these local geometries must be different and hopefully unique for each locality of the data set. Since the plan coordinates usually follow a regular pattern (i.e. they are usually represented by a grid structure with equal spacing between coordinate points) consequently, it becomes hard to differentiate small chunk of coordinates from one place to another in an indoor area. For instance, consider the indoor area represented by Figures 2 and 3 . Figure 2 shows the scenario of regular geometry of plan coordinates. Note here that the two structures 1 and 2, indicated in the figure, are difficult to differentiate. To resolve this issue and create uniqueness throughout the coordinate plan local geometries, the use of a very small number of fingerprinted readings (as low as 1\%) is proposed to create a perturbation in such geometries. In other words, the fingerprinted data is responsible only for introducing such uniqueness throughout the coordinate plan localities. Figure 3 shows the idea of geometry perturbation. The bold red dots in the figure represent the fingerprinted data. These points act as neighbors for all other points in the indoor area. The dotted and colored arrows show distance of the blue squares (or picked up elements from a set) to the fingerprints. The discrete distance vector formed from these distances is unique for every element. So, by comparing these figures, it can be inferred that each grid point can be represented uniquely in the spatial domain. If the geometry

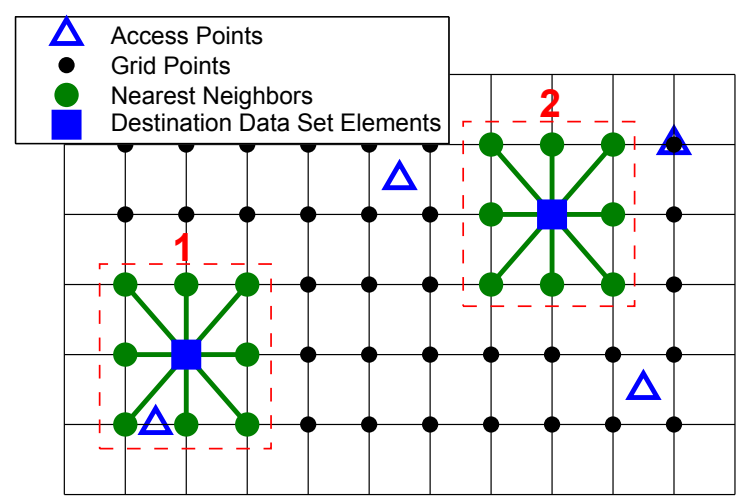

Fig. 2. Regular geometry of the indoor plan coordinates. Because of the regular geometry the two structures 1 and 2 are very similar. So the blue squares (or elements in the destination data set) cannot be identified uniquely.

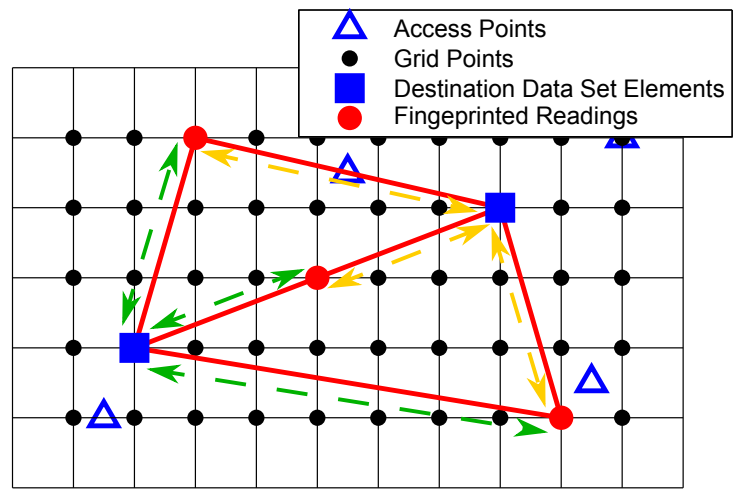

Fig. 3. Perturbed Geometry of the indoor plan coordinates. Based on the distance of blue squares to the red dots, the blue squares (or elements in the destination data set) can be uniquely identified.

perturbation is not taken into account, then many false matches will occur, which results in high localization errors. That is, the two elements (indicated by blue squares) taken from the destination data set shown in Figure 2 cannot be identified uniquely since they have same local geometries. This is illustrated in Section 5. So, the fingerprinted data (RSSs and their coordinates) is introduced as first $f$ data points in both the source and destination data sets. The remaining readings in set $\mathcal{X}$ consist of crowd sourced readings and localization requests and the remaining elements in set $\mathcal{Z}$ are the remaining coordinates of the indoor area. Let $\mathcal{X}_{r}$ and $\mathcal{Z}_{r}$ represent the remaining elements in sets $\mathcal{X}$ and $\mathcal{Z}$, respectively. These are given as $\mathcal{X}_{r}=\left\{\mathbf{x}_{f+1}, \mathbf{x}_{f+2}, \cdots, \mathbf{x}_{M}\right\}$ and $\mathcal{Z}_{r}=\left\{\mathbf{z}_{f+1}, \mathbf{z}_{f+2}, \cdots, \mathbf{z}_{N}\right\}$.

As described earlier, to create the necessary geometry perturbation required for correct matching of geometries, the first $f$ calibration elements are used as neighbors for all the other elements in the respective sets and discrete distance vectors are obtained. For the $i^{t h}$ element in set $\mathcal{X}_{r}$, the discrete distance vector obtained is as follows

$$
\mathbf{e}_{i}^{\mathcal{X}_{r}}=\left[\begin{array}{llll}
0 & e_{i 1} & \cdots & e_{i f}
\end{array}\right]^{T}, \quad i=1,2, \cdots, M-f
$$


where, the first entry represents the Euclidean distance of the $i^{t h}$ element to itself, which is equal to 0 , and the remaining entries represent the distance to the first $f$ elements (calibration readings or fingerprints) in set $\mathcal{X}$. This is depicted in Figure 3. The unlabeled data (crowd sourced readings or localization requests) are represented by blue squares and the fingerprints by red circles.

Similarly, the discrete distance vectors are obtained for all the elements in set $\mathcal{Z}_{r}$, which for the $j^{\text {th }}$ element in this set, can be represented as

$$
\mathbf{e}_{j}^{\mathcal{Z}_{r}}=\left[\begin{array}{llll}
0 & e_{j 1} & \cdots & e_{j f}
\end{array}\right]^{T}, \quad j=1,2, \cdots, N-f
$$

\subsection{Matching of Local Geometries}

One very recent method to represent and match local geometries in unsupervised manifold alignment is the use of spline curves. These spline curves can be used to match local geometries as follows. The spline curves (which are continuous parameterized curves) are fitted to the discrete distance vectors $\mathbf{e}_{i}^{\mathcal{X}_{r}}$ and $\mathbf{e}_{j}^{\mathcal{Z}_{r}}$, which are defined in (9) and (10), respectively. So there are $(M-f)$ curves in the source data set $\mathcal{X}_{r}$ and $(N-f)$ curves in destination data set $\mathcal{Z}_{r}$. The closeness of the local geometry of one element in the source data set with that of the other element in destination data set is measured by the enveloped area and gradient between the curves. Define $g_{i}^{\mathcal{X}_{r}}(u)$ and $g_{j}^{\mathcal{Z}_{r}}(u)$ as the curves fitted to the discrete distance vectors corresponding to the $i^{\text {th }}$ element in set $\mathcal{X}_{r}$ and the $j^{\text {th }}$ element in set $\mathcal{Z}_{r}$, respectively, using cubic spline interpolation [26]. The curves defined by $g_{i}^{\mathcal{X}_{r}}(u)^{\prime}$ 's are normalized $^{3}$ to 1 . Similarly, the process is repeated for the curves defined by $g_{j}^{\mathcal{Z}_{r}}(u)^{\prime}$ 's.

Now, define $\nabla g_{i}^{\mathcal{X}_{r}}(u)$ and $\nabla g_{j}^{\mathcal{Z}_{r}}(u)$ the gradients of $g_{i}^{\mathcal{X}_{r}}(u)$ and $g_{j}^{\mathcal{Z}_{r}}(u)$, respectively. We can thus define $\mathbf{H}$ as the local geometry similarity matrix, whose $i-j$ 's element $h_{i j}$ (referred to as the local geometry similarity indicator) is given by

$h_{i j}=\int_{0}^{f}\left(\left|g_{i}^{\mathcal{X}_{r}}(u)-g_{j}^{\mathcal{Z}_{r}}(u)\right|+\beta\left|\nabla g_{i}^{\mathcal{X}_{r}}(u)-\nabla g_{j}^{\mathcal{Z}_{r}}(u)\right|\right) d u$

where $\beta$ is a balancing constant and $u$ is the integration variable. The integration is solved by the Composite Simpson's rule [26]. Note that, from the definition of $h_{i j}$ we can infer that the smaller the $h_{i j}$ the larger the similarity between the two data points $\mathbf{x}_{i}$ and $\mathbf{z}_{j}$. Now we can use the similarity indicator $h_{i j}$ to find the best matching between $\mathcal{X}_{r}$ and $\mathcal{Z}_{r}$ and hence create the local geometry matching set $\mathcal{D}$ as the set of pairs as follows

$$
\mathcal{D}=\left\{\left(\mathbf{x}_{i}, \mathbf{z}_{j}\right) \mid h_{i j}=\min _{1 \leq t \leq N-f} h_{i t}, \text { and } h_{i j}<\eta\right\}
$$

In other words, the data point $\mathbf{z}_{j}$ from set $\mathcal{Z}_{r}$ is considered to be matched with point $\mathbf{x}_{i}$ from set $\mathcal{X}_{r}$, and thus are added as a matched pair $\left(\mathbf{x}_{i}, \mathbf{z}_{j}\right)$ to $\mathcal{D}$, if it has the largest similarity

3. This is because the RSS readings are higher dimensional as compared to the coordinates. When the discrete distance vectors are obtained from the elements in the RSS readings data set, the distance values are larger as compared to those obtained from the elements in the coordinates data set. Normalizing to 1 helps to obtain good closeness measure between the elements from these two sets. (i.e. smallest $h_{i j}$ ) among all other points in $\mathcal{Z}_{r}$ and $h_{i j}$ is below a certain threshold $\eta$. In this matching set two points $\mathbf{x}_{i}$ and $\mathbf{x}_{i}{ }^{\prime}$ might be matched to the same coordinate $\mathbf{z}_{j}$ and some $\mathbf{x}_{i}$ 's might not get any matches.

\subsection{Manifold Alignment}

After obtaining some matched pairs in set $\mathcal{D}$, we have two types of elements in the source set $\mathcal{X}_{r}$, namely ones that are matched to elements in destination set $\mathcal{Z}$ and hence belong to $\mathcal{D}$ and ones that are not and hence do not belong to $\mathcal{D}$. So we have to give high weight to these matched pairs in the alignment formulation as will be described shortly. The preservation of local geometries is also essential while aligning data in a lower dimensional space. Taking these facts into account, the objective function for the unsupervised manifold alignment formulation consists of three terms $J_{d}$, $J_{f}$ and $J_{s}$, which are responsible for minimizing the intermanifold distance, local geometry matching between the data sets and preservation of local geometries within the data sets, respectively.

Consider an affine transformation matrix $\boldsymbol{\alpha}_{i}$, which is assigned to the element $\mathbf{x}_{i}$ in $\mathcal{X}_{r}$. The matrix $\boldsymbol{\alpha}_{i}$ is of dimension $2 \times R$, where 2 is the dimension of the coordinates and $R$ represents the number of access points, that is, the dimensionality of the element in the source (RSS readings) data set $\mathcal{X}$. The term $J_{d}$ contains elements of the set $\mathcal{X}_{r}$, which are not present in the local geometry matching set $\mathcal{D}$ and is expressed as

$$
J_{d}=\sum_{\mathbf{x}_{i} \in \mathcal{X}_{r},\left(\mathbf{x}_{i}, \mathbf{z}_{j}\right) \notin \mathcal{D}} \min _{\mathbf{z}_{j} \in \mathcal{Z}}\left\|\boldsymbol{\alpha}_{i} \mathbf{x}_{i}-\mathbf{z}_{j}\right\|_{2}^{2}
$$

Let $\mathbf{z}_{j}$ be the element in $\mathcal{Z}$ that gives the lowest intermanifold distance for $\mathbf{x}_{i}$. Thus, we can write

$$
\begin{aligned}
J_{d}=\sum_{\mathbf{x}_{i} \in \mathcal{X}_{r},\left(\mathbf{x}_{i}, \mathbf{z}_{j}\right) \notin \mathcal{D}}\left(\mathbf{x}_{i}^{T} \boldsymbol{\alpha}_{i}^{T} \boldsymbol{\alpha}_{i} \mathbf{x}_{i}-\mathbf{x}_{i}^{T} \boldsymbol{\alpha}_{i}^{T} \mathbf{z}_{j}\right. \\
\left.-\mathbf{z}_{j}^{T} \boldsymbol{\alpha}_{i} \mathbf{x}_{i}+\mathbf{z}_{j}^{T} \mathbf{z}_{j}\right)
\end{aligned}
$$

Let $\nabla J_{d}$ denote the gradient of $J_{d}$ with respect to $\boldsymbol{\alpha}\left(\frac{\partial J_{d}}{\partial \boldsymbol{\alpha}_{i}}\right)$, i.e.

$$
\nabla J_{d}=\sum_{\mathbf{x}_{i} \in \mathcal{X}_{r},\left(\mathbf{x}_{i}, \mathbf{z}_{j}\right) \notin \mathcal{D}}\left(2 \boldsymbol{\alpha}_{i} \mathbf{x}_{i} \mathbf{x}_{i}^{T}-2 \mathbf{z}_{j} \mathbf{x}_{i}^{T}\right)
$$

The term $J_{f}$ contains elements of set $\mathcal{X}_{r}$, which are present in the local geometry matching set $\mathcal{D}$ and is expressed as

$$
J_{f}=\sum_{\left(\mathbf{x}_{i}, \mathbf{z}_{j}\right) \in \mathcal{D}}\left\|\boldsymbol{\alpha}_{i} \mathbf{x}_{i}-\mathbf{z}_{j}\right\|_{2}^{2} w_{i j}
$$

where $w_{i j}$ is the weight computed between $\mathbf{x}_{i}$ and $\mathbf{z}_{j}$ by heat kernel i.e. $w_{i j}=e^{-\frac{h_{i j}^{2}}{\gamma}}$ (the choice of $\gamma$ is described in Section 5). Expanding the summand of $J_{f}$ as done previously, yields

$$
\begin{array}{r}
J_{f}=\sum_{\left(\mathbf{x}_{i}, \mathbf{z}_{j}\right) \in \mathcal{D}}\left(\mathbf{x}_{i}^{T} \boldsymbol{\alpha}_{i}^{T} \boldsymbol{\alpha}_{i} \mathbf{x}_{i}-\mathbf{x}_{i}^{T} \boldsymbol{\alpha}_{i}^{T} \mathbf{z}_{j}\right. \\
\left.-\mathbf{z}_{j}^{T} \boldsymbol{\alpha}_{i} \mathbf{x}_{i}+\mathbf{z}_{j}^{T} \mathbf{z}_{j}\right) w_{i j}
\end{array}
$$

The corresponding gradient is given by

$$
\nabla J_{f}=\sum_{\left(\mathbf{x}_{i}, \mathbf{z}_{j}\right) \in \mathcal{D}}\left(2 \boldsymbol{\alpha}_{i} \mathbf{x}_{i} \mathbf{x}_{i}^{T}-2 \mathbf{z}_{j} \mathbf{x}_{i}^{T}\right) w_{i j}
$$


For preservation of local geometries, neighboring information is taken into account. So the crowd sourced information together with the localization requests serve the purpose by getting $k$ nearest neighbors ( $\mathrm{knn}$ ) among them for the point $\mathbf{x}_{i}$. The last term, $J_{s}$, is responsible for preservation of local geometries. This is expressed as

$$
J_{s}=\sum_{\mathbf{x}_{i} \in \mathcal{X}_{r}} \sum_{\mathbf{x}_{j} \in \operatorname{knn}\left(\mathbf{x}_{i}\right)}\left\|\boldsymbol{\alpha}_{i}-\boldsymbol{\alpha}_{j}\right\|_{F}^{2}
$$

where $\operatorname{knn}\left(\mathbf{x}_{i}\right)$ is the set of the $k$ nearest neighbors of $\mathbf{x}_{i}$. Minimizing this term results in minimizing the distances of the mappings of the $k$ nearest neighbors of $\mathbf{x}_{i}$ to its own mapping. Expanding $J_{s}$ yields

$J_{s}=\sum_{\mathbf{x}_{i} \in \mathcal{X}_{r}} \sum_{\mathbf{x}_{j} \in \operatorname{knn}\left(\mathbf{x}_{i}\right)} \operatorname{Tr}\left(\boldsymbol{\alpha}_{i} \boldsymbol{\alpha}_{i}^{T}-\boldsymbol{\alpha}_{i} \boldsymbol{\alpha}_{j}^{T}-\boldsymbol{\alpha}_{j} \boldsymbol{\alpha}_{i}^{T}+\boldsymbol{\alpha}_{j} \boldsymbol{\alpha}_{j}^{T}\right)$

and its gradient is

$$
\nabla J_{s}=\sum_{\mathbf{x}_{i} \in \mathcal{X}_{r}} \sum_{\mathbf{x}_{j} \in \operatorname{knn}\left(\mathbf{x}_{i}\right)}\left(2 \boldsymbol{\alpha}_{i}-2 \boldsymbol{\alpha}_{j}\right)
$$

The overall objective function to be minimized is thus the combination of the above mentioned three terms

$$
J=\mu_{d} J_{d}+\mu_{f} J_{f}+\mu_{s} J_{s}
$$

and its gradient is represented as

$$
\nabla J=\mu_{d} \nabla J_{d}+\mu_{f} \nabla J_{f}+\mu_{s} \nabla J_{s}
$$

where $\mu_{d}, \mu_{f}$ and $\mu_{s}$ are weighting factors for their respective terms, which are chosen empirically. Here $\mu_{f}$ is given the largest weight to stress on the matching of local geometries and $\mu_{d}$ is given the least weight.

The Quasi-Newton BFGS algorithm [27] is employed to solve this nonlinear optimization problem. The objective function to be minimized is supplied to the Quasi-Newton BFGS algorithm along with the gradients. The optimized values of mappings $\boldsymbol{\alpha}_{i}$ 's are obtained. These mappings then transform the elements in the source (or RSS readings) data set to the destination (or coordinates) data set. The best matchings are then obtained by a mapping function, which computes the closest point pairs.

\subsection{Localization Algorithm}

Here the geometry perturbed unsupervised manifold alignment algorithm is applied to localize users using approximately $1 \%$ calibration readings, some crowd sourced readings, localization requests and plan coordinates of the indoor area. The goal is to learn the mappings $\boldsymbol{\alpha}_{i}$ 's described previously. The data sets follow the assumed correlation pattern, that is, neighboring points have stronger correlation as compared to distant points. The localization algorithm consists of the following steps:

1) Build the source and destination data sets as defined in (4) and (5) respectively.

2) Obtain the spline curve for each element in sets $\mathcal{X}$ and $\mathcal{Z}$ except for the first $f$ elements as described in Section 4.

3) Obtain the local geometry similarity matrix $\mathbf{H}$ by using (11).
4) Set up the cost function as described in (22) and then apply the Quasi-Newton BFGS algorithm to obtain the optimized transformation matrices, $\boldsymbol{\alpha}_{i}{ }^{\prime}$ s.

5) Estimate the positions for localization requests (last $r$ elements in set $\mathcal{X}$ ) by computing the mapping function $\phi\left(\mathbf{x}_{i}\right)$,

$$
\boldsymbol{\phi}\left(\mathbf{x}_{i}\right)=\underset{\mathbf{z}_{j} \in \mathcal{Z}}{\arg \min }\left\|\boldsymbol{\alpha}_{i} \mathbf{x}_{i}-\mathbf{z}_{j}\right\|_{2}
$$

$\mathbf{z}_{j}$, for which $\phi\left(\mathbf{x}_{i}\right)$ is minimum, represents the closest match. This, in fact, is the estimated location, $\hat{\mathbf{p}}_{l_{i}}$ (see (6)).

The computational complexity of cubic spline interpolation is $O((f+1)+\log (f+1))$ in one dimension. The computational complexity for each iteration of BFGS is $O\left((2 R(o+r))^{2}\right)$. The convergence rate of BFGS algorithm is super linear.

\section{TESting Results for PROPOSEd INDOOR LO- CALIZATION FRAMEWORK}

This section describes the testing of the proposed indoor localization framework using real measurements from the $4^{\text {th }}$ floor of Bahen Center, at University of Toronto, depicted in Figure 4, which is the same indoor plan used in [28], [29]. The black dots represent the 219 plan coordinates considered in this indoor environment. The distance between each two neighboring points is $1 \mathrm{~m}$. This indoor environment is used for comparison of the results with the previously proposed semi-supervised solutions.

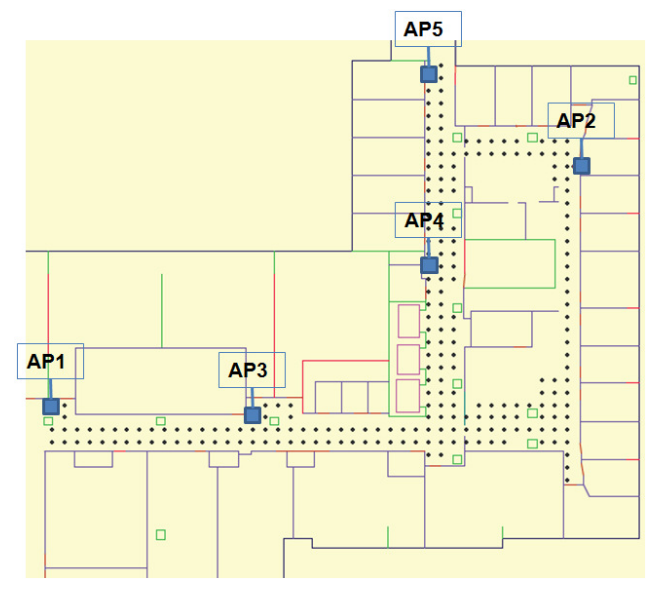

Fig. 4. Floor plan of the considered indoor area

The data collection is done in the same way as described in [28], [29]. For testing the algorithm as scenario independent, the crowd sourced readings and localization requests are chosen randomly throughout the indoor environment. Only set $\mathcal{C}$ is obtained in the offline stage while rest of the readings (crowd sourced information and localization requests) are obtained in the online stage. The mean localization error (average error of all location estimations) is plotted against the variation of different parameters. The percentage variation shown for some parameters is obtained by normalizing against the total number of points in the indoor area. The curves are obtained by averaging over a large number of runs of the algorithm. The chosen value 
of $\beta$ is 0.1 for numerical integration. There is no need for manual adjustment of $\eta$. Its value is set at the lower $15 \%$ of the total values in matrix $\mathbf{H}$. The nominal weight $\left(\mu_{s}=1\right)$ is given to the term preserving local geometries within each data set. The largest weight $\left(\mu_{f}=10\right)$ is given to the term minimizing local geometry matching between the data sets because we are exploiting the underlying correlation pattern of the data sets. And the smallest weight $\left(\mu_{s}=0.1\right)$ is given to the term minimizing the inter-manifold distance. The choice of $\gamma$ (appearing in weight computation by heat kernel, $w_{i j}=e^{-\frac{h_{i j}^{2}}{\gamma}}$ ) is made by using $\gamma=-\frac{\eta^{2}}{\ln (0.9)}$, where $\eta$ is defined in (12). During each trial of the proposed algorithm the crowd sourced readings and localization requests are selected randomly. The random selections cause the similarities between matched pairs to change each time. This means $\eta$ will change during each trial. So $\gamma$ is made dependent on $\eta$ and selected such that all the matched pairs fall within $10 \%$ decay of the heat kernel.

For comparisons while testing, following methodologies are considered:

- The proposed unsupervised manifold alignment algorithm with geometry perturbation.

- Unsupervised manifold alignment algorithm without geometry perturbation.

- The raw semi-supervised algorithm proposed in [28], [29] which considers the fingerprinted readings and localization requests only and excludes the crowd sourced information.

- A modified version of the semi-supervised algorithm in [28], [29], where the crowd sourced readings are treated as localization requests.

- The interpolation method, proposed in [30], which makes use of radial basis functions. The estimated position is the function of RSS readings.

The following subsections show the effect of varying different parameters on the mean localization error.

\subsection{Localization Errors of Individual Runs}

Figure 5 depicts the localization error for individual runs of the methodologies described above for a fingerprinting load of approximately $1 \%$ (only 2 readings in our case). The crowd sourced readings are set to $10 \%$ and the localization requests are set to $7 \%$. The figure clearly shows that the performance of the proposed unsupervised technique with a minimal fingerprinting load for geometry perturbation is much better as compared to both variants of the semisupervised approaches in terms of the mean and variance of the localization error. The performance is also better as compared to the interpolation method as described in [30]. The results also show that the proposed geometry perturbation helps a lot in improving the performance as compared to the raw unsupervised approach. This proves the merits of the proposed indoor localization framework.

Table 1 summarizes the numerical comparison of the mean and variance of the reported error trends in Figure 5. Clearly, our proposed scheme with geometry perturbation significantly outperforms all other schemes at this very low level of fingerprinting load, both in the mean and variance of the localization error.

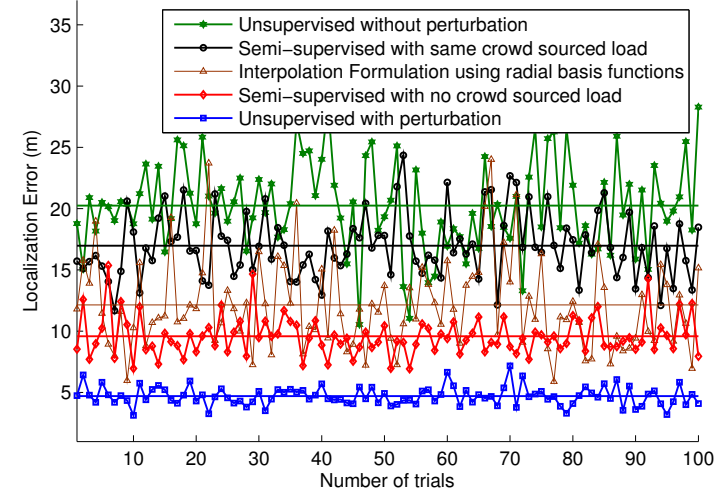

Fig. 5. Localization error against the number of algorithm runs

TABLE 1

Comparison with respect to mean and variance

\begin{tabular}{|l|c|c|}
\hline Algorithm & Mean (m) & Variance \\
\hline Unsupervised without perturbation & 20.24 & 13.45 \\
\hline Semi-supervised with same crowd sourced load & 16.98 & 7.04 \\
\hline Interpolation Method proposed in [30] & 12.14 & 13.69 \\
\hline Semi-supervised with no crowd sourced load & 9.58 & 2.53 \\
\hline Unsupervised with perturbation & 4.7 & 0.58 \\
\hline
\end{tabular}

\subsection{Variation of Calibration Readings (fingerprinting load)}

Figure 6 shows the performance comparison between the proposed algorithm and the raw and modified semisupervised algorithms against the variation of the percentage of fingerprinting (i.e. calibration) load. The crowd sourced information is set to $20 \%$ and localization requests are set to $7 \%$. A first important observation from the figure is that the performance of our proposed unsupervised scheme is not affected by the amount of calibration load from $1 \%$ to $5 \%$. This is clearly due to the fact that the calibration data, whether $1 \%$ or up to $5 \%$, is not used in the main alignment and location estimation processes. They are only used in our proposed scheme to perturb the local geometries. In that matter, only few points are enough to make this perturbation and adding more points would not result in any improvement.

The performance is also compared with the interpolation method considered in [30]. The comparison shows a much better performance for the proposed algorithm at low percentage of fingerprints as depicted in Figure 6. However, the raw semi-supervised approach and interpolation method of [30] dominates at relatively higher percentage of fingerprinting data, which is expected. There is very less degradation in performance at $15 \%$ of the fingerprinting load as compared to $100 \%$ for these techniques [29], [30]. This does not demerit the proposed indoor localization framework since it is designed to operate at very low level $(1 \%)$ of fingerprinting load. Around $60 \%$ performance improvement is observed at this low level of fingerprinting load as compared to semisupervised techniques. 


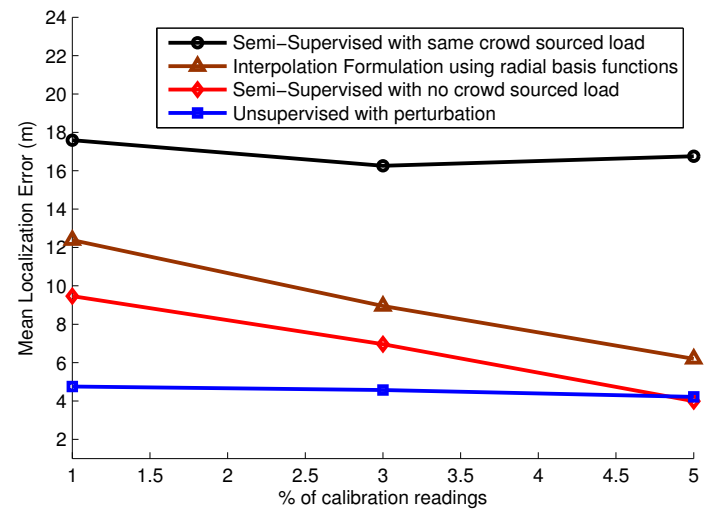

Fig. 6. Mean localization error against the percentage of calibration readings

\subsection{Variation of Crowd Sourced Information}

Figure 7 shows the mean localization error plotted against the increasing percentage of crowd sourced readings for the proposed unsupervised scheme with geometry perturbation and the modified semi-supervised algorithm. The semi-supervised algorithm is modified such that the crowd sourced readings are included in unlabeled data. Almost 1\% fingerprinted readings are selected and the localization is done for 15 requests ( $7 \%$ of the total number of grid points). It can be observed that the error remains approximately stable for both algorithms as the percentage of crowd sourced information is increased. This is usually expected since increasing the unlabeled data in unsupervised learning does not usually improve the performance. This result is very important as it means that the proposed unsupervised algorithm does not need to wait for collecting a large number of crowd sourced readings before performing localization. This allows the use of real-time crowd sourced readings (i.e. readings collected at the same time or very short time before the localization requests) for localization. This has an added advantage that the temporal variations in RSS values, which occur due to dynamic nature of the indoor environment, are taken care of to a great extent.

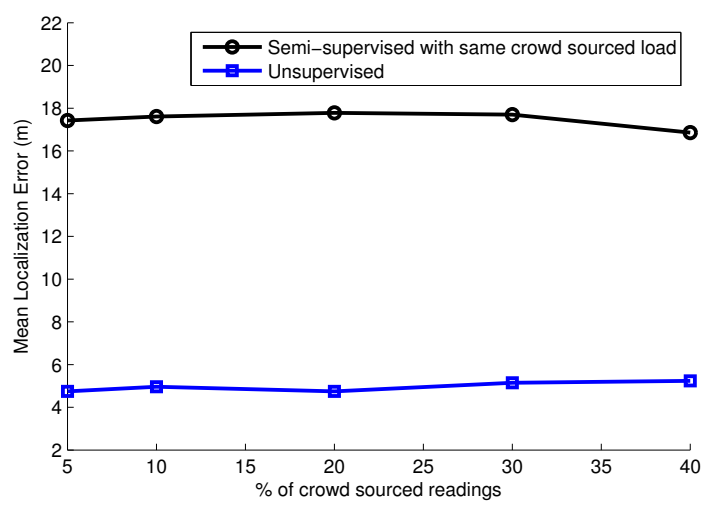

Fig. 7. Comparison of unsupervised and semi-supervised algorithms with same crowd sourced information

\subsection{Variation of Localization Requests}

Figure 8 shows the mean localization error plotted against the increasing percentage of localization requests. The fingerprinted readings considered here are again set to less than $1 \%$ and the crowd sourced information is set to $10 \%$. It can be observed from the figure that the mean localization error remains almost stable. This is again due to the fact that increasing the unlabeled data (here localization requests) in unsupervised manifold alignment does not usually result in performance improvement. The same trend can also be observed for the semi-supervised approaches. The result obtained from this figure is again very important as it shows that the proposed indoor localization framework does not need to collect a large number of localization requests to achieve better performance. Whether it is one localization request or many, the performance remains stable.

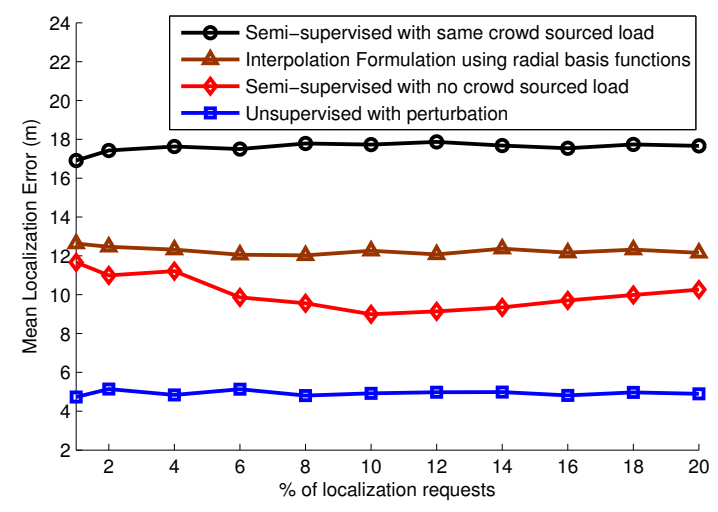

Fig. 8. Mean localization error against the percentage of localization requests

The crowd sourced readings and localization requests considered in Figures 7 and 8 can be related to blue squares shown in Figure 3. The discrete distance vectors (equation (9)) are obtained for each of these readings as shown in the figure. The red dots in the figure represent the fingerprints. Rest of the procedure follows as explained in Subsections 4.2 and 4.3 .

\section{Radio Map Estimation}

This section describes the estimation of the complete radio map of the indoor environment. With few calibration points and few localization requests we have enough data to obtain the whole radio map of the indoor area. This is described in the following.

\subsection{Estimation Problem Setup}

The data required for estimating the radio map consists of few calibration readings or fingerprints, few localization requests with corresponding position estimates, which are obtained from the localization algorithm, and plan coordinates of the indoor area. We assume that the fingerprints and plan coordinates are already available. When the system is deployed in the indoor environment, the localization requests are put up during normal operation of the system. The position estimates for these localization requests are obtained as indicated by (6). 
The continuous running of the indoor localization algorithm provides the position estimates for the localization requests. After successive iterations of the algorithm, the RSS readings get accumulated at some positions. The accumulated RSS readings are averaged at those positions after fixed number of iterations. So, we have few positions with calibration readings (fingerprints) and few positions with averaged RSS readings. Note that the averaged RSS readings at corresponding positions may be different from the actual fingerprints at those positions. The remaining positions do not contain any RSS readings and thus the RSS values are estimated at those positions. Figure 9 represents the scenario of gathering RSS readings after fixed number of iterations of the indoor localization algorithm. The averaged

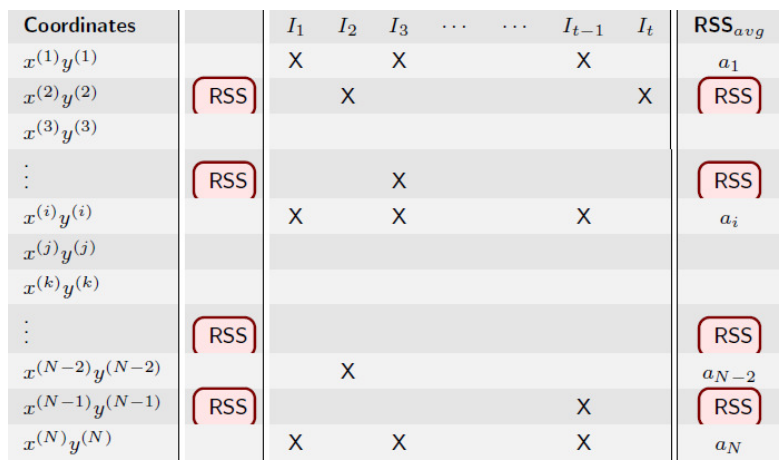

Fig. 9. Data collection through continuous running of the indoor localization algorithm. The highlighted RSSs represent the fingerprints. The symbols ' $X$ ' represent the RSS readings (localization requests), which are put during successive iterations. These all are $R$ dimensional vectors. The number of iterations shown here are $I_{t}$.

RSS readings are appended to the calibration readings set $\mathcal{C}$. So, the calibration readings and averaged RSS readings with corresponding coordinates are treated as labeled data in the estimation problem. Let the set $\Psi$ represent this labeled information. This is given as

$\boldsymbol{\Psi}=\left\{\left[\begin{array}{c}c_{1}^{(1)} \\ c_{2}^{(1)} \\ \vdots \\ c_{R}^{(1)}\end{array}\right], \cdots,\left[\begin{array}{c}c_{1}^{(f)} \\ c_{2}^{(f)} \\ \vdots \\ c_{R}^{(f)}\end{array}\right],\left[\begin{array}{c}a_{1}^{(1)} \\ a_{2}^{(1)} \\ \vdots \\ a_{R}^{(1)}\end{array}\right], \cdots,\left[\begin{array}{c}a_{1}^{(b)} \\ a_{2}^{(b)} \\ \vdots \\ a_{R}^{(b)}\end{array}\right]\right\}$

The first $f$ elements in set $\boldsymbol{\Psi}$ represent the fingerprints and next $b$ elements represent the averaged RSS readings. The cardinality of this set is $(f+b)$, where $(f+b) \ll N$.

The coordinates corresponding to the RSS readings in set $\Psi$ are given in set $\mathcal{P}_{\Psi}$, i.e., $\mathcal{P}_{\Psi}=$ $\left\{\mathbf{p}_{c_{1}}, \cdots, \mathbf{p}_{c_{f}}, \hat{\mathbf{p}}_{a_{1}}, \cdots, \hat{\mathbf{p}}_{a_{b}}\right\}$. The sequence of the elements in set $\mathcal{P}_{\Psi}$ is same as appearing in set $\boldsymbol{\Psi}$, that is, the first $f$ elements are the corresponding coordinates of the calibration readings and next $b$ elements are the positions where accumulated RSS readings are averaged.

From the sets $\Psi$ and $\mathcal{P}_{\Psi}$, this can be inferred that the RSS readings are known at the labeled $(f+b)$ positions. Now, we need to estimate the RSS values at remaining positions of the indoor environment. The estimation is done by considering RSS readings from one AP at a time. So, the RSS readings from the $k^{\text {th }}$ AP can be divided into two column vectors, $\mathbf{v}_{k}$, the vector of unknown RSS values and $\mathbf{u}_{k}$, the vector of known RSS values. The labeled RSS values corresponding to the $k^{t h} \mathrm{AP}$, picked up from set $\boldsymbol{\Psi}$, are given as

$$
\mathbf{u}_{k}=\left[\begin{array}{llllll}
c_{k}^{(1)} & \cdots & c_{k}^{(f)} & a_{k}^{(1)} & \cdots & a_{k}^{(b)}
\end{array}\right]^{T}
$$

The size of vector $\mathbf{u}_{k}$ is $(f+b) \times 1$. There are $(N-f-b)$ remaining positions where RSS values corresponding to the $k^{t h}$ AP are to be determined. So, the size of vector $\mathbf{v}_{k}$ is $(N-f-b) \times 1$. The estimation problem is thus

$$
\hat{\mathbf{v}}_{k}=E\left[\mathbf{v}_{k} \mid \mathbf{u}_{k}\right]
$$

Since the solution of conditional expectation given in the above equation is hard to get in closed form so the linear least-mean-squares estimator [24] is used to solve it. This is described as follows.

Consider RSS values from the $k^{\text {th }} \mathrm{AP}$, the linear leastmean-squares estimator of $\mathbf{v}_{k}$ given $\mathbf{u}_{k}$ is given as

$$
\hat{\mathbf{v}}_{k}=\mathbf{R}_{v_{k} u_{k}} \mathbf{R}_{u_{k}}^{-1} \mathbf{u}_{k}
$$

where, $\mathbf{R}_{v_{k} u_{k}}$ and $\mathbf{R}_{u_{k}}$ are the covariance matrices. These matrices correspond to the readings taken from the $k^{\text {th }}$ AP.

Once the matrices $\mathbf{R}_{v_{k} u_{k}}$ and $\mathbf{R}_{u_{k}}$ are known, the RSS values at the remaining positions can be determined. Now, the main task is to determine $\mathbf{R}_{v_{k} u_{k}}$ and $\mathbf{R}_{u_{k}}$. The information available to us is the labeled data given by (25), their corresponding coordinates given by set $\mathcal{P}_{\Psi}$ and the remaining coordinates of the indoor area. The readings and coordinates data sets we used in unsupervised manifold alignment problem have the same underlying correlation pattern. Note that the points closer to each other have stronger correlation as compared to distant points. Taking this fact into account, we will approximate these covariances using two methods which we will describe next.

The first method does this approximation by heat kernel (HK) and the second one by Locally Linear Embedding (LLE) [22]. For the heat kernel method, the closer the points are to each other, the higher the weight assigned to them. Similarly, the LLE preserves neighborhood correlation between the points while moving them to lower dimensional space. The matrix $\mathbf{R}_{v_{k} u_{k}}$ represents the relationship between unknown values in vector $\mathbf{v}_{k}$ and known values in vector $\mathbf{u}_{k}$. To obtain this relationship, the coordinates of the indoor plan are partitioned into two sets i.e. one set contains the coordinates corresponding to labeled data as given by set $\mathcal{P}_{\Psi}$ and the second set contains the coordinates, where RSS values are to be determined. The coordinates in the second set are, in fact, the remaining coordinates of the indoor plan and are accumulated in set $\mathcal{Q}$.

$$
\mathcal{Q}=\left\{\mathbf{q}_{1}, \mathbf{q}_{2}, \cdots, \mathbf{q}_{N-f-b}\right\}
$$

$\mathbf{R}_{u_{k}}$ represents the relationship between the knowns, that is, RSS values corresponding to the $k^{t h} \mathrm{AP}$ as indicated by the vector $\mathbf{u}_{k}$ in (26). Following subsections describe the approaches to approximate the matrices $\mathbf{R}_{v_{k} u_{k}}$ and $\mathbf{R}_{u_{k}}$.

\subsection{Approximation by Heat Kernel}

The heat kernel calculates the weight between $i^{t h}$ and $j^{t h}$ elements by $w_{i j}=e^{-\frac{d_{i j}^{2}}{\gamma}}$, where $d_{i j}$ is the Euclidean distance between the $i^{t h}$ and $j^{t h}$ elements. The elements belong to 
the same set when approximating $\mathbf{R}_{u_{k}}$ and to different sets in case of $\mathbf{R}_{v_{k} u_{k}}$. The value of $\gamma$ chosen here is such that all the elements fall within $10 \%$ of the maximum Euclidean distance $d_{i j}$ computed between the elements.

\subsubsection{Approximation of $\mathbf{R}_{v_{k} u_{k}}$}

The Euclidean distance, $d_{i j}$, is computed between the coordinates. The $i^{\text {th }}$ coordinate pair is taken from set $\mathcal{Q}$ while the $j^{\text {th }}$ coordinate pair is taken from set $\mathcal{P}_{\Psi}$. The heat kernel defined above then computes the weight between the unknown and known elements. The entry $w_{i j}^{v_{k} u_{k}}$ is the $i j^{t h}$ entry of the matrix $\mathbf{R}_{v_{k} u_{k}}$ and this matrix is given as

$\mathbf{R}_{v_{k} u_{k}}=\left[\begin{array}{cccc}w_{11}^{v_{k} u_{k}} & w_{12}^{v_{k} u_{k}} & \cdots & w_{1(f+b)}^{v_{k} u_{k}} \\ w_{21}^{v_{k} u_{k}} & w_{22}^{v_{k} u_{k}} & \cdots & w_{2(f+b)}^{v_{k} u_{k}} \\ \vdots & \vdots & \ddots & \vdots \\ w_{(N-f-b) 1}^{v_{k} u_{k}} & w_{(N-f-b) 2}^{v_{k} u_{k}} & \cdots & w_{(N-f-b)(f+b)}^{v_{k} u_{k}}\end{array}\right]$

The size of this matrix is $(N-f-b) \times(f+b)$, which corresponds to the number of elements in $\mathbf{v}_{k}$ and $\mathbf{u}_{k}$.

\subsubsection{Approximation of $\mathbf{R}_{u_{k}}$}

The Euclidean distance of each element in vector $\mathbf{u}_{k}$ is computed with all the elements in $\mathbf{u}_{k}$. The individual entries of $\mathbf{R}_{u_{k}}$ are then computed from the heat kernel defined earlier. The size of the matrix $\mathbf{R}_{u_{k}}$ is $(f+b) \times(f+b)$ and it is given as

$$
\mathbf{R}_{u_{k}}=\left[\begin{array}{cccc}
w_{11}^{u_{k}} & w_{12}^{u_{k}} & \cdots & w_{1(f+b)}^{u_{k}} \\
w_{21}^{u_{k}} & w_{22}^{u_{k}} & \cdots & w_{2(f+b)}^{u_{k}} \\
\vdots & \vdots & \ddots & \vdots \\
w_{(f+b) 1}^{u_{k}} & w_{(f+b) 2}^{u_{k}} & \cdots & w_{(f+b)(f+b)}^{u_{k}}
\end{array}\right]
$$

As the localization requests from users are obtained randomly in the indoor area, so the matrix $\mathbf{R}_{u_{k}}$, formed in (31) from the labeled elements, becomes singular sometimes. The regularization term is added to $\mathbf{R}_{u_{k}}$ to account for the singularity, that is, $\mathbf{R}_{u_{k}}=\mathbf{R}_{u_{k}}+\epsilon \mathbf{I}_{(f+b)}$, where $\epsilon$ is a regularization factor and $\mathbf{I}_{(f+b)}$ is an $(f+b) \times(f+b)$ identity matrix.

This gives us approximations of the covariance matrices for the $k^{\text {th }}$ AP. After approximating $\mathbf{R}_{v_{k} u_{k}}$ and $\mathbf{R}_{u_{k}}$, the linear least-mean-squares estimator given by (28) is used to estimate RSS values at all the positions other than the labeled positions.

The above process is repeated to get these approximations for all the $R$ APs present in the indoor environment. The RSS readings estimated at a grid point from all the APs are then stacked as a vector to represent the estimated fingerprint at that point. So the estimated RSS values (estimated fingerprints) for all the $(N-f-b)$ grid points can be represented by the set $\Theta$, i.e.

$$
\boldsymbol{\Theta}=\left\{\left[\begin{array}{c}
\hat{v}_{1}^{(1)} \\
\hat{v}_{2}^{(1)} \\
\vdots \\
\hat{v}_{R}^{(1)}
\end{array}\right],\left[\begin{array}{c}
\hat{v}_{1}^{(2)} \\
\hat{v}_{2}^{(2)} \\
\vdots \\
\hat{v}_{R}^{(2)}
\end{array}\right], \cdots,\left[\begin{array}{c}
\hat{v}_{1}^{(N-f-b)} \\
\hat{v}_{2}^{(N-f-b)} \\
\vdots \\
\hat{v}_{R}^{(N-f-b)}
\end{array}\right]\right\}
$$

or, in compact form as $\boldsymbol{\Theta}=\left\{\boldsymbol{\theta}_{1}, \boldsymbol{\theta}_{2}, \cdots, \boldsymbol{\theta}_{N-f-b}\right\}$, where $\boldsymbol{\theta}_{i}$ represents the estimated fingerprint at the $i^{\text {th }}$ grid point.

\subsection{Approximation by Locally Linear Embedding}

The second method considered for estimating the covariance matrices makes use of weight computation by Locally Linear Embedding (LLE) [22]. Out of several dimensionality reduction techniques, the LLE is used here since it strongly preserves the correlation between the element and its neighbors. This is consistent with our data sets since they have the same underlying correlation pattern. Instead of using the nearest neighbors, the labeled data indicated by set $\mathcal{P}_{\Psi}$ and (26) are used as neighbors for all the elements while approximating the covariance matrices. This labeled data include RSS values in set $\boldsymbol{\Psi}$ and corresponding coordinates in set $\mathcal{P}_{\Psi}$. These act as neighbors while computing $\mathbf{R}_{v_{k} u_{k}}$ and $\mathbf{R}_{u_{k}}$. The procedure of obtaining the approximation of these matrices is described in the following.

\subsubsection{Approximation of $\mathbf{R}_{v_{k} u_{k}}$}

For $\mathbf{R}_{v_{k} u_{k}}$, all the elements in set $\mathcal{P}_{\Psi}$ are used as neighbors for each element present in set $\mathcal{Q}$. The LLE then computes weight of the element $\mathbf{q}_{i}$ in set $\mathcal{Q}$ with all its neighbors in set $\mathcal{P}_{\Psi}$ by the following relation

$$
\begin{aligned}
\min _{w_{i j}^{v_{k} u_{k}} \forall \mathbf{p}_{j} \in \mathcal{P}_{\Psi}}\left|\mathbf{q}_{i}-\sum_{\mathbf{p}_{j} \in \mathcal{P}_{\Psi}} w_{i j}^{v_{k} u_{k}} \mathbf{p}_{j}\right|^{2} \\
\text { s.t. } \quad \sum_{\mathbf{p}_{j} \in \mathcal{P}_{\Psi}} w_{i j}^{v_{k} u_{k}}=1
\end{aligned}
$$

where, $w_{i j}^{v_{k} u_{k}}$ is the weight computed by LLE between the $i^{\text {th }}$ and $j^{\text {th }}$ elements in sets $\mathcal{P}_{\Psi}$ and $\mathcal{Q}$, respectively. This, in fact, is the $i j^{t h}$ entry of the matrix $\mathbf{R}_{v_{k} u_{k}}$. As mentioned in the previous subsection, the size of this matrix is $(N-f-b) \times(f+b)$. The structure of this matrix is the same as shown by (30) except for the fact that weights here are computed by LLE instead of heat kernels.

\subsubsection{Approximation of $\mathbf{R}_{u_{k}}$}

For approximating $\mathbf{R}_{u_{k}}$, each element in vector $\mathbf{u}_{k}$ uses all other elements in the same vector for computing weights by LLE, that is, for the $i^{\text {th }}$ element in vector $\mathbf{u}_{k}$, the remaining $(f+b)-1$ elements act as its neighbors. The weights $w_{i j}^{u_{k}}$ 's are given by the following relation

$$
\begin{aligned}
& \min _{w_{i j}^{u_{k}} \forall u_{k}(j) \in \mathbf{u}_{k} \wedge(j \neq i)}\left|u_{k}(i)-\sum_{u_{k}(j) \in \mathbf{u}_{k} \wedge(j \neq i)} w_{i j}^{u_{k}} u_{k}(j)\right|^{2} \\
& \text { s.t. } \quad \sum_{u_{k}(j) \in \mathbf{u}_{k} \wedge(j \neq i)} w_{i j}^{u_{k}}=1
\end{aligned}
$$

The weight $w_{i j}^{u_{k}}$ is the $i j^{t h}$ entry of the matrix $\mathbf{R}_{u_{k}}$. As mentioned earlier, the size of this matrix is $(f+b) \times(f+b)$. The structure of this matrix is same as shown by (31) with the only difference that here the weights are computed by LLE.

As mentioned previously, the matrix $\mathbf{R}_{u_{k}}$ becomes singular most of the times due to gathering of localization requests randomly throughout the indoor environment. So, the regularization term is added here also to account for the singularity in matrix $\mathbf{R}_{u_{k}}$. The RSS estimates for all the positions indicated in set $\mathcal{Q}$ are obtained by using linear least-mean-squares estimator given by (28). 
The approximations for the matrices obtained above are used for estimating RSS readings corresponding to the $k^{t h}$ AP. The above process is repeated for all the APs present in the indoor environment. So, the estimated RSS values from all the APs at a grid point represent the estimated fingerprint at that point. The estimated fingerprints are represented in the same fashion as shown in (32).

\section{Testing Results for Radio Map Estima- TION}

This section describes the performance testing of the proposed solution for radio map estimation. As mentioned earlier, the radio map construction relies on the data obtained from the indoor localization framework proposed earlier. This data includes few calibration readings, few localization requests with corresponding position estimates and plan coordinates of the indoor area.

The indoor floor plan considered is the same as shown in Figure 4. The initial running of the indoor localization algorithm provides estimated positions for few localization requests, which help in estimating the radio map later. For performance evaluation, the root mean square (rms) error is plotted by comparing the actual fingerprints with the estimated one. The rms error calculation does not include the calibration readings (fingerprints) and is given as

$$
\text { rmserr }=\sqrt{\frac{\sum_{i=1}^{b}\left(\mathbf{a}_{i}-\mathbf{c}_{\hat{p}_{a_{i}}}\right)^{2}+\sum_{i=1}^{N-f-b}\left(\boldsymbol{\theta}_{i}-\mathbf{c}_{i}\right)^{2}}{R \times(N-f)}}
$$

The $b$ elements in first summation represent the few localization requests gathered during initial running of the algorithm. $\mathbf{a}_{i}$ represents the averaged RSS readings at the $i^{t h}$ grid point and $\mathbf{c}_{\hat{p}_{a}}$ represents the actual fingerprint at the same grid point. Similarly, for the second summation in (35), $\boldsymbol{\theta}_{i}$ represents the estimated fingerprint at the $i^{t h}$ grid point and $\mathbf{c}_{i}$ the actual fingerprint at the same point. In the testing, comparison between semi-supervised and unsupervised algorithms is considered along with different parameter variations. The performance of the proposed solution is also checked by varying different parameters. The calibration readings and localization requests are randomly selected throughout the indoor area. The curves shown in the following are obtained by averaging over several runs of the algorithm.

\subsection{Effect of varying calibration readings}

Figure 10 shows the rms error plotted against the increasing percentage of calibration readings (fingerprints). The localization requests considered here are $1 \%$. The proposed indoor localization algorithm is run for fixed number of iterations ( 1 and 10 iterations shown here). The radio map estimation is done for both the semi-supervised algorithm in [28], [29] and unsupervised indoor localization framework proposed in this work. Figure 11 shows the similar curves but for approximation of the covariance matrices using LLE. It is important to note first that, unlike the localization algorithm, the radio map estimation performance using our proposed method, and for both LLE and HK, does improve with the increase of the calibration load. This comes very

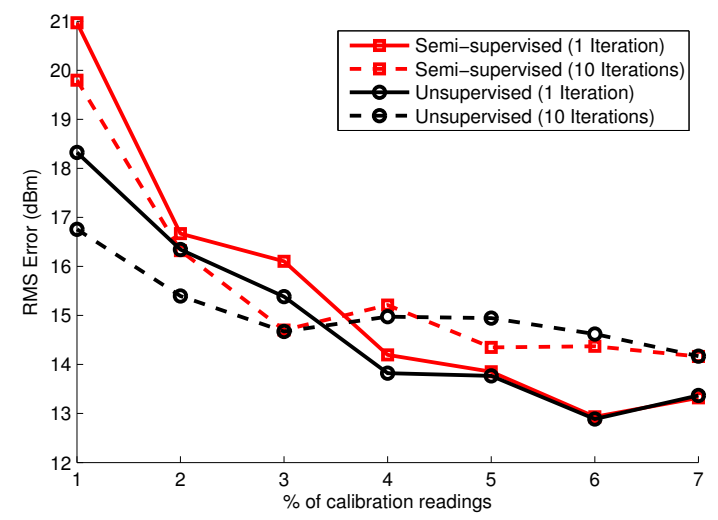

Fig. 10. RMS error against the percentage of calibration readings using HK approx. for covariance matrices

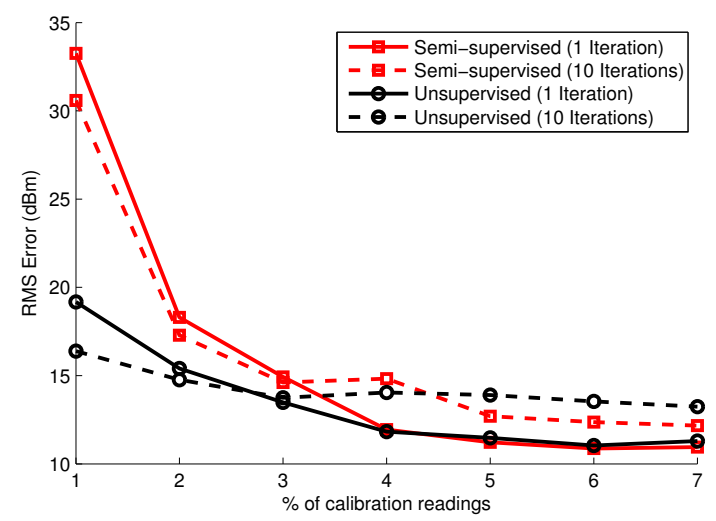

Fig. 11. RMS error against the percentage of calibration readings using LLE approx. for covariance matrices

naturally as a consequence of using the accurate calibration information in vector $\left(\mathbf{u}_{\mathbf{k}}\right)$ as part of the linear least-meansquares estimator in Equation (28). It is clear from the basics of linear least-mean-squares estimation that, the larger and more accurate this vector, the better the estimate.

From Figures 10 and 11, this can be observed that the proposed unsupervised algorithm shows huge improvement in performance as compared to the semi-supervised approach at only $1 \%$ of fingerprinting load, $1 \%$ of localization requests and 1 iteration of the algorithm. Further improvement is observed after 10 iterations of the algorithm. However, for 10 iterations of the algorithm the performance starts to deteriorate after $3 \%$ of the calibration readings. This corresponds to the total labeled data of $13 \%$, that is, almost 28 points out of total 219 points in the indoor environment considered here. This is consistent with the results and supports the fact that the proposed unsupervised algorithm is meant to operate at very low level of fingerprinting load. The same effect can also be observed by varying the localization requests while fixing the number of iterations to 1 . The $10 \%$ localization requests with 1 iteration is almost equivalent to $1 \%$ localization requests with 10 iterations since both of them will provide almost the same number of position estimates. The comparison of the figures also show the better performance of using LLE approximations rather 
than the heat kernel at low percentage of fingerprinting load.

\subsection{Effect of including localization requests}

Figure 12 shows the effect of including localization requests on rms error. The proposed unsupervised framework for

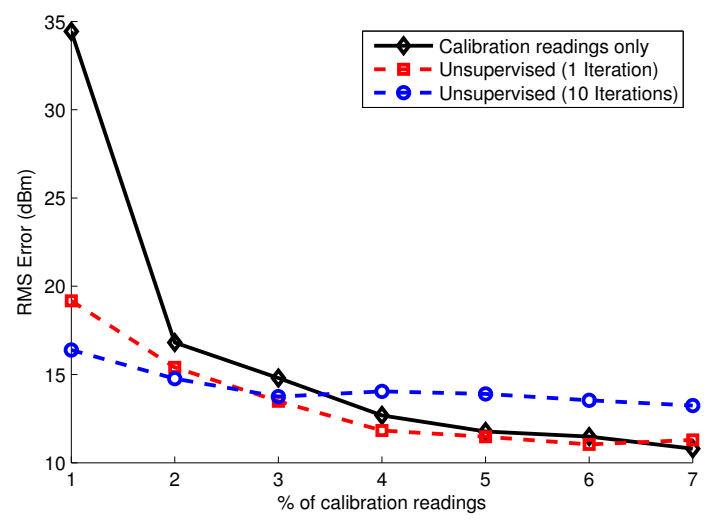

Fig. 12. Effect of including localization requests in radio map estimation. LLE aaproximation is used here

indoor localization is considered here for radio map estimation. The curves shown here use LLE approximations of the covariance matrices. The localization requests considered are $1 \%$. The algorithm is run for 1 and 10 iterations. Another curve is plotted, which does not take into account the localization requests and use only calibration readings to estimate the radio map. The comparison of the curves show considerable improvement in performance when localization requests are included as the labeled data for radio map estimation. The performance improvement is really high at $1 \%$ of the fingerprinting load. The percentage improvement in performance for 1 iteration and 10 iterations of the algorithm is $44 \%$ and $52 \%$, respectively, at $1 \%$ of the fingerprinting load.

\subsection{Effect of using actual fingerprints for labeled data at estimated positions}

The indoor localization algorithm outputs position estimates for the localization requests, which are already in some error. This leaves us with the question: How far we are in estimating the radio map if we are not using actual fingerprints at the concerned grid points? Figures 13 and 14 (using heat kernel and LLE approximations for covariance matrices respectively) show the comparison of including the localization requests and corresponding position estimates with that of the actual fingerprints at those estimated positions. The localization requests considered here again are $1 \%$ and the indoor localization algorithm is run for 1 iteration. The comparison of the curves show that we are not far away in rms error. The rms error, by not using actual fingerprints at concerned grid points, falls in between $1 \sim 2 \mathrm{dBm}$.

\subsection{Pictorial view of estimated RSS readings}

Figure 15 shows the actual RSS signal strengths measured from the first $\mathrm{AP}^{4}$. The bright colors in larger filled circles

4. The first AP is actually the AP 1 shown in Figure 4.

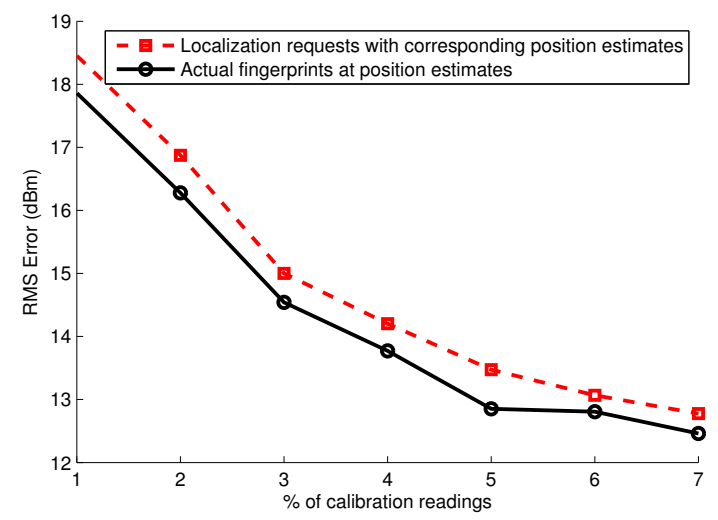

Fig. 13. Effect of using actual fingerprints for labeled data at estimated positions. HK approximation is used here

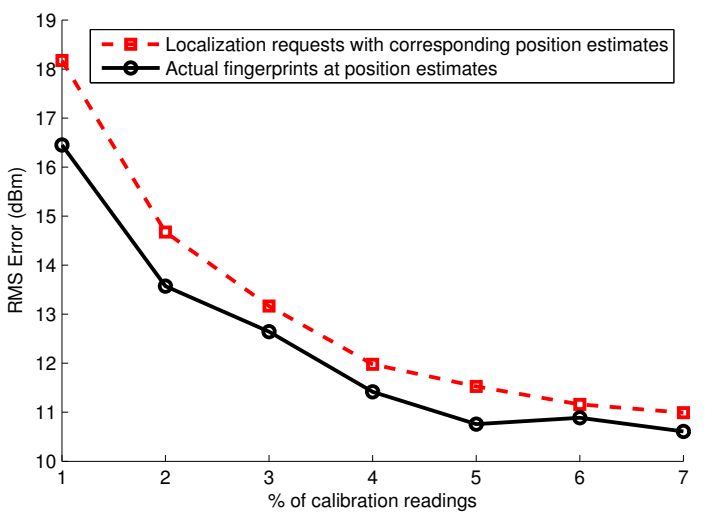

Fig. 14. Effect of using actual fingerprints for labeled data at estimated positions. LLE approximation is used here

show stronger signal strengths. The color darkens and size of the circle reduces with the decreasing signal strength. The minute dots around top right corner of the figure shows no coverage by AP 1 . The localization requests considered

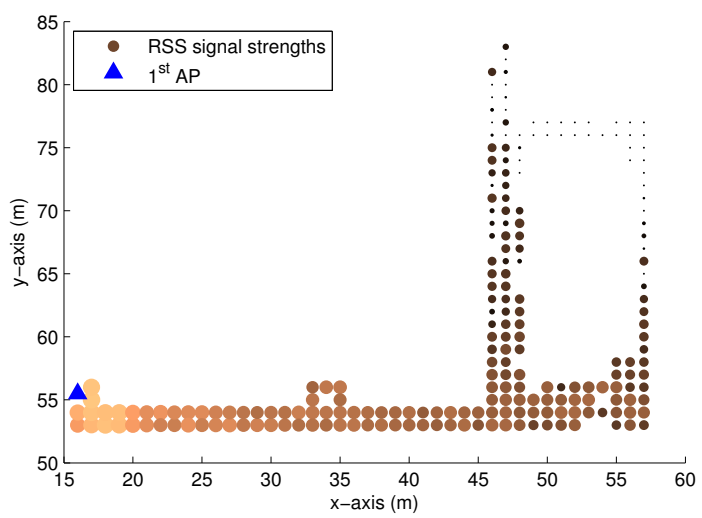

Fig. 15. Radio Map showing actual RSS signal strengths measured from AP 1

are $1 \%$ and the calibration readings (fingerprints) are also $1 \%$. The estimated RSS signal strengths shown here consider approximation of the covariance matrices by using LLE. The 
signal strength map obtained by 1 iteration of the indoor localization algorithm is shown in Figure 16. Figure 17

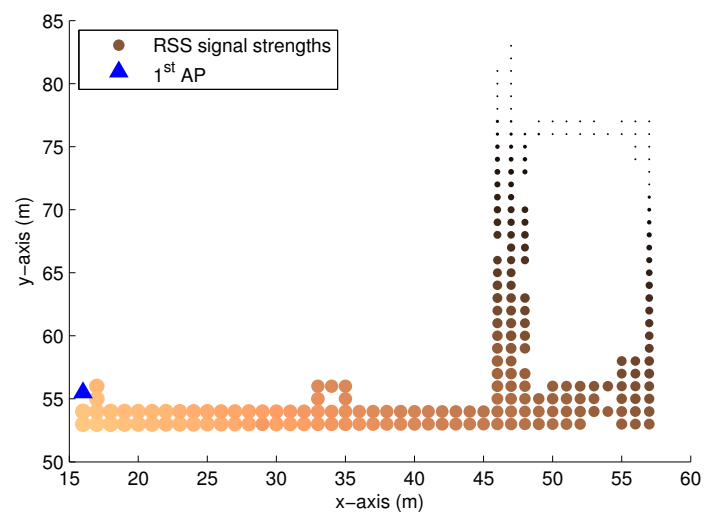

Fig. 16. Radio Map showing estimated RSS signal strengths for AP 1 $\left(I_{t}=1\right)$

shows the similar map but with 10 iterations of the indoor localization algorithm.

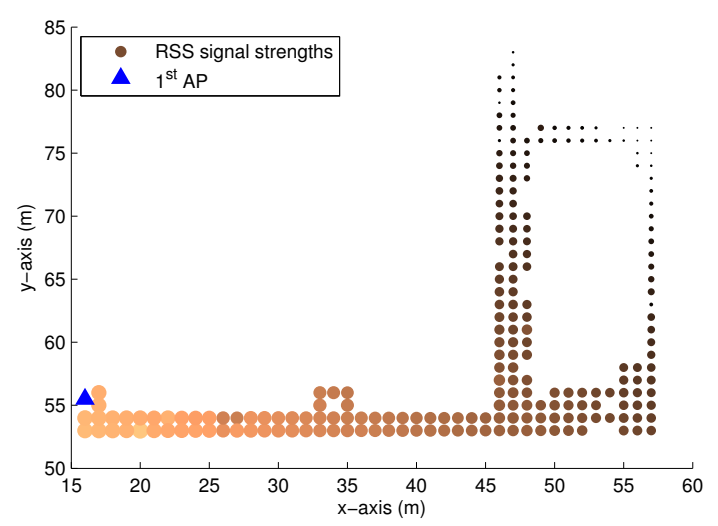

Fig. 17. Radio Map showing estimated RSS signal strengths for AP 1 $\left(I_{t}=10\right)$

\section{Conclusion}

The proposed indoor localization framework using unsupervised manifold alignment with geometry perturbation almost eliminates the workload required for practical deployment of such systems. The collection of very small number of fingerprints together with some crowd sourced readings, localization requests and indoor plan coordinates help in location sensing. The remarkable performance is achieved at very small number of calibration points (approximately $1 \%$ ) as compared to semi-supervised approaches. Due to the reduction in fingerprinting load (almost 99\%), the practical deployment of proposed indoor localization system in a new indoor environment does not require any initial setup time. Secondly, the few collected fingerprints together with few localization requests, treated as labeled data, help to estimate the complete radio map of the indoor environment instantaneously. The inclusion of localization requests for radio map estimation shows almost $50 \%$ performance improvement as compared to using only fingerprints.
The comparison of using actual fingerprints instead of localization requests accumulated at the estimated positions shows that we are doing only $1 \sim 2 \mathrm{dBm}$ worse.

\section{REFERENCES}

[1] K. Majeed, S. Sorour, T. Y. Al-Naffouri, and S. Valaee, "Indoor localization using unsupervised manifold alignment with geometry perturbation," in IEEE WCNC14 Track 3 (Mobile and Wireless Networks) (IEEE WCNC14 Track 3 : NET), Istanbul, Turkey, Apr. 2014, pp. 2994-2999.

[2] H. Liu, H. Darabi, P. Banerjee, and J. Liu, "Survey of Wireless Indoor Positioning Techniques and Systems," IEEE Transactions on Systems, Man and Cybernetics, Part C (Applications and Reviews), vol. 37, no. 6, pp. 1067-1080, Nov. 2007.

[3] S. Gezici and H. V. Poor, "Position Estimation via Ultra-Wide-Band Signals," Proceedings of the IEEE, vol. 97, no. 2, pp. 386-403, Feb. 2009.

[4] S. Gezici, "A Survey on Wireless Position Estimation," Wireless Personal Communications, vol. 44, no. 3, pp. 263-282, Feb. 2008.

[5] G. Shen, Z. Chen, P. Zhang, T. Moscibroda, and Y. Zhang, "Walkiemarkie: indoor pathway mapping made easy," in Proc. of USENIX NSDI, 2013.

[6] A. Rai, K. K. Chintalapudi, V. N. Padmanabhan, and R. Sen, "Zee: zero-effort crowdsourcing for indoor localization," in Proceedings of the 18th annual international conference on Mobile computing and networking, 2012, pp. 293-304.

[7] H. Wang, S. Sen, A. Elgohary, M. Farid, M. Youssef, and R. R. Choudhury, "No need to war-drive: unsupervised indoor localization," in Proceedings of the 10th international conference on Mobile systems, applications, and services, 2012, pp. 197-210.

[8] Z. Yang, C. Wu, and Y. Liu, "Locating in fingerprint space: wireless indoor localization with little human intervention," in Proceedings of the 18th annual international conference on Mobile computing and networking, 2012, pp. 269-280.

[9] P. Bahl and V. N. Padmanabhan, "RADAR: An in-building RFbased user location and tracking system," in INFOCOM 2000. Nineteenth Annual Joint Conference of the IEEE Computer and Communications Societies. Proceedings. IEEE, 2000, vol. 2, pp. 775784.

[10] C. Feng, W. S. A. Au, S. Valaee, and Z. Tan, "Compressive sensing based positioning using RSS of WLAN access points," in INFOCOM, 2010 Proceedings IEEE, 2010, pp. 1-9.

[11] C. Feng, W. Au, S. Valaee, and Z. Tan, "Orientation-aware indoor localization using affinity propagation and compressive sensing," in Computational Advances in Multi-Sensor Adaptive Processing (CAMSAP), 2009 3rd IEEE International Workshop on, 2009, pp. 261-264.

[12] C. Feng, W. S. A. Au, S. Valaee, and Z. Tan, "Received-SignalStrength-Based Indoor Positioning Using Compressive Sensing," IEEE Transactions on Mobile Computing, vol. 11, no. 12, pp. 19831993, Dec. 2012

[13] A. Kushki, K. N. Plataniotis, and A. N. Venetsanopoulos, "KernelBased Positioning in Wireless Local Area Networks," IEEE Transactions on Mobile Computing, vol. 6, no. 6, pp. 689-705, Jun. 2007.

[14] G. Sun, J. Chen, W. Guo, and K. R. Liu, "Signal processing techniques in network-aided positioning: a survey of state-of-theart positioning designs," Signal Processing Magazine, IEEE, vol. 22, no. 4 , pp. 12-23, 2005.

[15] R. Singh, L. Macchi, C. S. Regazzoni, and K. N. Plataniotis, "A statistical modelling based location determination method using fusion technique in WLAN," Proc. IEEE IWWAN, 2005.

[16] Hadi Jamali-Rad, Hamid Ramezani, Geert Leus, Sparsity-aware multi-source RSS localization, Signal Processing, Volume 101, August 2014, Pages 174-191, ISSN 0165-1684.

[17] H. Jamali-Rad and G. Leus, "Sparsity-Aware Multi-Source TDOA Localization," IEEE Transactions on Signal Processing, vol. 61, no. 19, pp. 4874-4887, Oct. 2013.

[18] C. Rizos, A. G. Dempster, B. Li, and J. Salter, "Indoor positioning techniques based on Wireless LAN," 2007.

[19] R. G. Baraniuk, "Compressive sensing [lecture notes]," Signal Processing Magazine, IEEE, vol. 24, no. 4, pp. 118-121, 2007.

[20] G. Kutyniok, "Compressed sensing: Theory and applications," CoRR, vol. abs/1203.3815, 2012.

[21] E. J. Candes and M. B. Wakin, "An Introduction To Compressive Sampling," IEEE Signal Processing Magazine, vol. 25, no. 2, pp. 21-30, Mar. 2008. 
[22] S. T. Roweis, Nonlinear Dimensionality Reduction by Locally Linear Embedding, Science, vol. 290, no. 5500, pp. 2323-2326, Dec. 2000.

[23] J. Ham, D. Lee, and L. Saul, Semisupervised alignment of manifolds, in Proceedings of the Annual Conference on Uncertainty in Artificial Intelligence, Z. Ghahramani and R. Cowell, Eds, 2005, vol. 10 , pp. 120-127.

[24] A. Sayed, Adaptive Filters, Wiley, 2008.

[25] Yuru Pei, Fengchun Huang, Fuhao Shi, and Hongbin Zha, Unsupervised Image Matching Based on Manifold Alignment, IEEE Transactions on Pattern Analysis and Machine Intelligence, vol. 34, no. 8, pp. 1658-1664, Aug. 2012.

[26] R.L. Burden and J.D. Faires, Numerical Analysis, seventh ed. Brookes Cole, 2000.

[27] J. Nocedal and S. Wright, Numerical Optimization, ser. Springer series in operations research and financial engineering. Springer 1999.

[28] S. Sorour, Y. Lostanlen, and S. Valaee, "RSS based indoor localization with limited deployment load.," in GLOBECOM, 2012, pp. 303-308.

[29] Sorour, S.; Lostanlen, Y.; Valaee, S.; Majeed, K., "Joint Indoor Localization and Radio Map Construction with Limited Deployment Load," Mobile Computing, IEEE Transactions on , vol.14, no.5, pp.1031-1043, May 12015

[30] J. Krumm and J. Platt, "Minimizing calibration efforts for an indoor 802.11 device location measurement system," Microsoft Research, MSR-TR-2003-82, Nov 2003.

[31] Xiaoyong Chai; Qiang Yang, "Reducing the Calibration Effort for Location Estimation Using Unlabeled Samples," Pervasive Computing and Communications, 2005. PerCom 2005. Third IEEE International Conference on , vol., no., pp.95-104, 8-12 March 2005

[32] Xiaoyong Chai; Qiang Yang, "Reducing the Calibration Effort for Probabilistic Indoor Location Estimation," Mobile Computing, IEEE Transactions on , vol.6, no.6, pp.649-662, June 2007

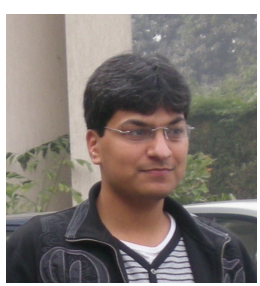

Khaqan Majeed received the MS degree in Electrical Engineering from King Fahd University of Petroleum and Minerals (KFUPM), Dhahran, Saudi Arabia in 2014 and the BSc degree (first division with honors) in Electrical Engineering from University of Engineering and Technology (UET), Lahore, Pakistan in 2009. He was a Lab Engineer cum Lecturer at the University of Management and Technology (UMT), Lahore, Pakistan until April 2011 and a Research Associate in High Performance Computing and Networking Lab (HPCNL) at Al-Khawarizmi Institute of Computer Science (Al-KICS UET), Lahore, Pakistan until January 2012. His research interests lie in the areas of indoor localization, machine learning, 2-Dimensional Direction-of-Arrival (DOA) estimation, and biomedical signal processing.

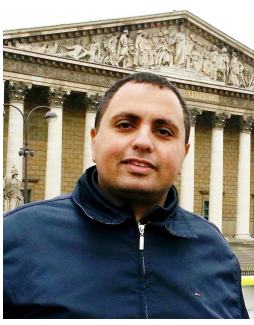

Sameh Sorour (S '98, M '11, S '15) received his B.Sc. and M.Sc. degrees in Electrical Engineering from Alexandria University, Egypt, in 2002 and 2006, respectively. In 2002, he joined the Department of Electrical Engineering, Alexandria University, where he was a Teaching and Research Assistant for three years and was promoted to Assistant Lecturer in 2006. In 2011, he obtained his Ph.D. degree from the Wireless and Internet Research Laboratory (WIRLab), Department of Electrical and Computer Engineering, University of Toronto, Canada. After two postdoctoral fellowships at University of Toronto and King Abduallah University of Science and Technology (KAUST), he joined KFUPM in September 2013. His research interests include network coding, indoor localization, coded cooperative data exchange for machine-to-machine communications, distributed cloud storage and storage area networks, online coded caching and smart networks.

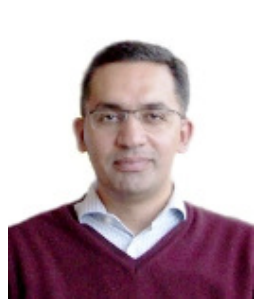

Tareq Y. Al-Naffouri received the B.S. degrees in mathematics and electrical engineering (with first honors) from King Fahd University of Petroleum and Minerals, Dhahran, Saudi Arabia, in 1994, the M.S. degree in electrical engineering from the Georgia Institute of Technology, Atlanta, in 1998, and the Ph.D. degree in electrical engineering from Stanford University, Stanford, $\mathrm{CA}$, in 2004. He was a visiting scholar at California Institute of Technology, Pasadena, CA, from January to August 2005 and during summer 2006. He was a Fulbright scholar at the University of Southern California from February to September 2008. He has held internship positions at NEC Research Labs, Tokyo, Japan, in 1998, Adaptive Systems Lab, University of California at Los Angeles in 1999, National Semiconductor, Santa Clara, CA, in 2001 and 2002, and Beceem Communications Santa Clara, CA, in 2004. He is currently an Associate Professor at the Electrical Engineering Department, King Abdullah University of Science and Technology (KAUST). His research interests lie in the areas of adaptive, statistical, and sparse signal processing and their applications to wireless communications, UWB, indoor localization, and sensor networks. He is also interested in the use of stochastic geometry for the analysis and design of wireless networks. He has over 120 publications in journal and conference proceedings, 9 standard contributions, 10 issued patents, and 4 pending. Dr. Al-Naffouri is the recipient of a 2001 Best Student Paper Award at the IEEE-EURASIP Workshop on Nonlinear Signal and Image Processing (NSIP) 2001 for his work on adaptive filtering analysis, the IEEE Education Society Chapter Achievement Award in 2008, and Al-Marai Award for innovative research in communication in 2009. Dr. Al-Naffouri has also been serving as an Associate Editor of Transactions on Signal Processing since August 2013.

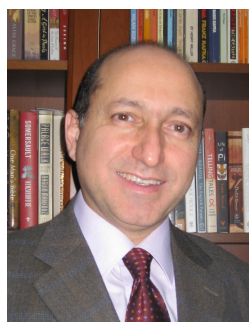

Shahrokh Valaee is with the Edward S. Rogers Sr. Department of Electrical and Computer Engineering, University of Toronto, where he is a Professor and the Associate Chair for Undergraduate Studies. He is the Founder and the Director of the Wireless and Internet Research Laboratory (WIRLab) at the University of Toronto. Professor Valaee was the TPC Co-Chair and the Local Organization Chair of the IEEE Personal Mobile Indoor Radio Communication (PIMRC) Symposium 2011. He was a Track Co-Chair of WCNC 2014 and the TPC Co-Chair of ICT 2015. He has been the guest editor for various journals. From December 2010 to December 2012 , he was the Associate Editor of the IEEE Signal Processing Letters, and an Editor of IEEE Transactions on Wireless Communications from 2010 to 2015 . He is currently an Editor of Elsevier Journal of Computer and System Science. Professor Valaee is a Fellow of the Engineering Institute of Canada. 